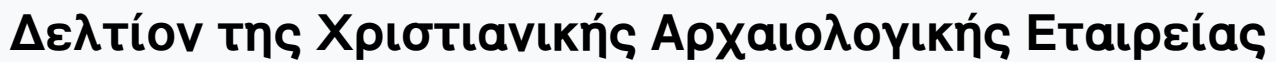

Tó 28 (2007)

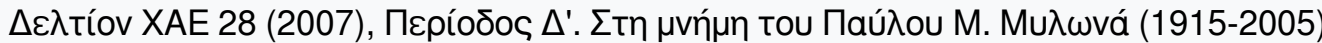

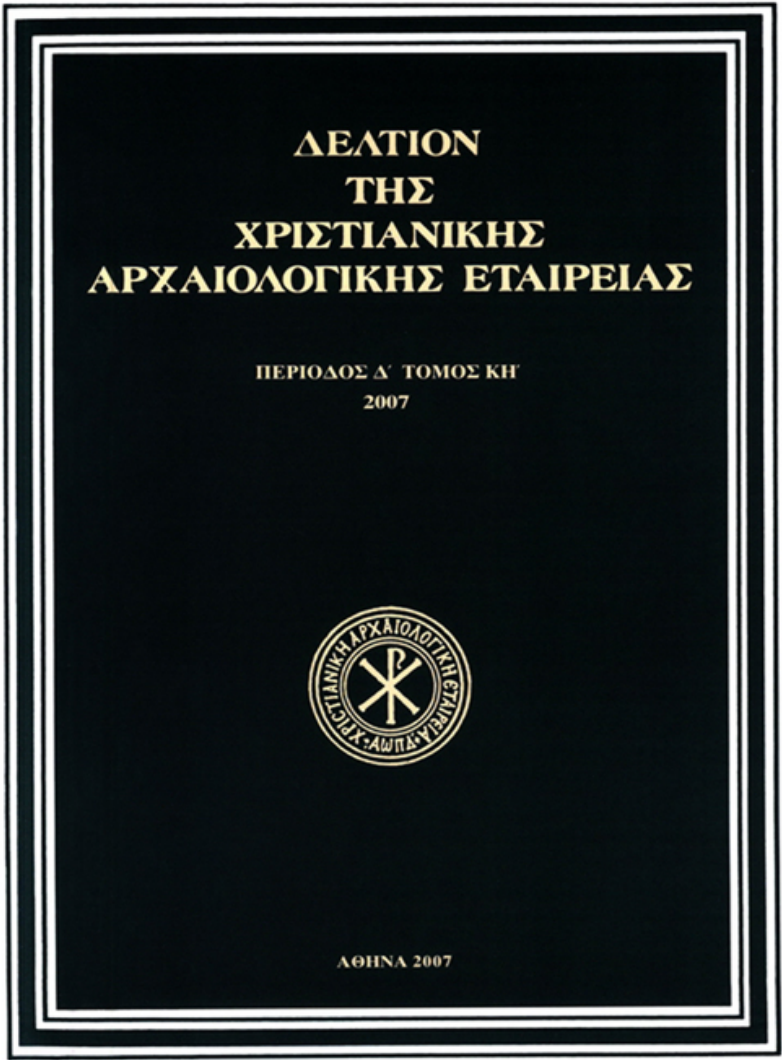

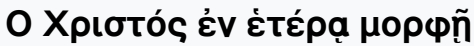

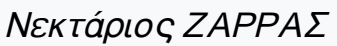

doi: $\underline{10.12681 / \text { dchae.588 }}$

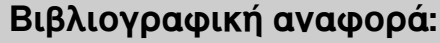

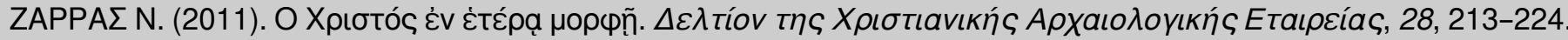
https://doi.org/10.12681/dchae.588 


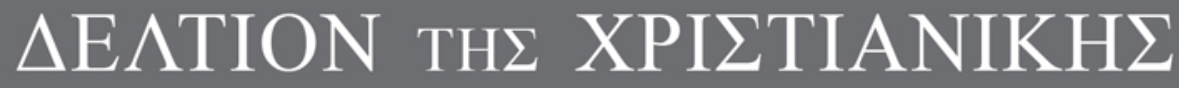 APXAIO $\Lambda$ ОГКН $\Sigma$ ETAIPEIA $\Sigma$}

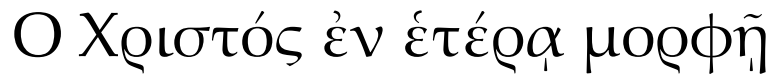

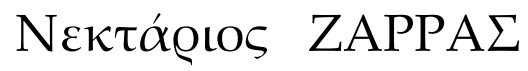

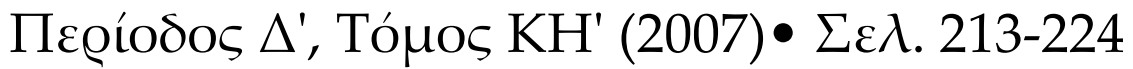
A@HNA 2007 


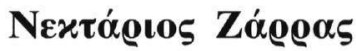

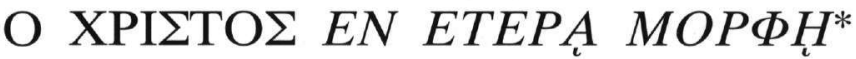

$\sum$

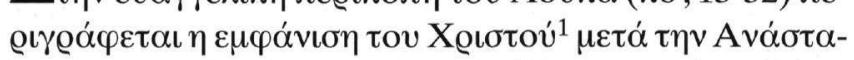

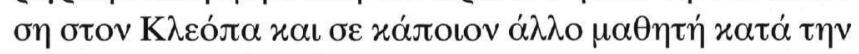

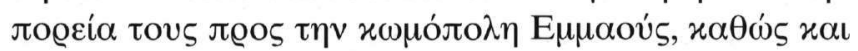

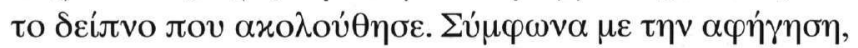

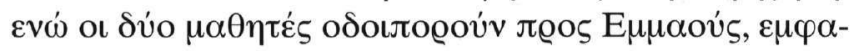

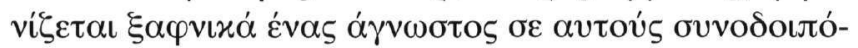

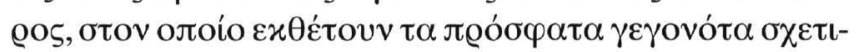

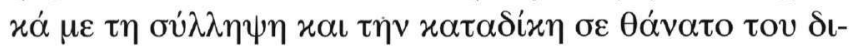

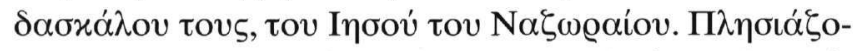

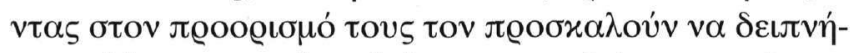

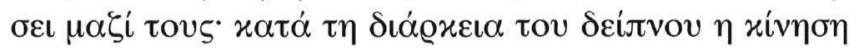

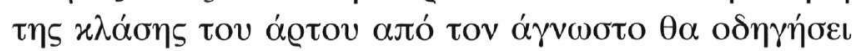

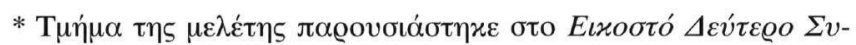

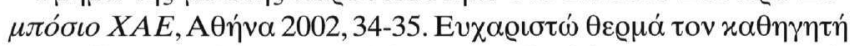

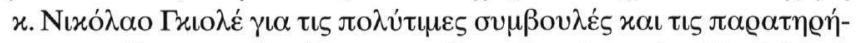

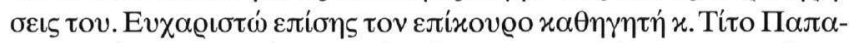

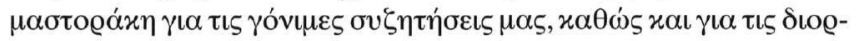

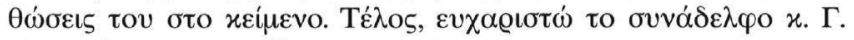

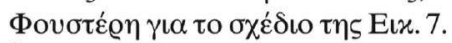

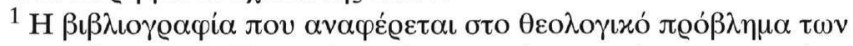

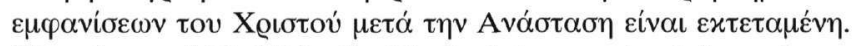
$\mathrm{B} \lambda$. $\varepsilon v \delta \varepsilon \iota \varkappa \tau \iota x \alpha \dot{\alpha}$ W. Michaelis, Die Erscheinungen des Auferstandenen, B $\alpha \sigma i \lambda \varepsilon i \alpha$ 1944. C. H. Dodd, The Appearances of the Risen Christ: An Essay in Form-Criticism of the Gospels, «Essays in Memory of R. H.

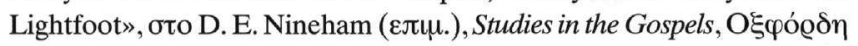
1957, 9-35. H. E. Von Campenhausen, Der Ablauf der Ostereignisse und das leere Grab (Sitzungsberichte der Heidelberg Akademie der Wissen-

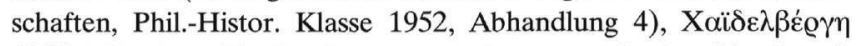
1966. J. E. Alsup, The Post-Ressurection Appearance Stories of the Gospel-

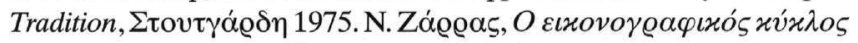

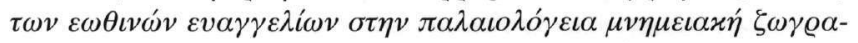

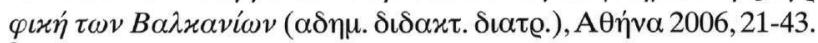

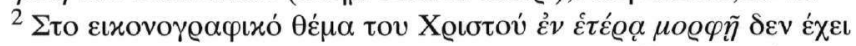

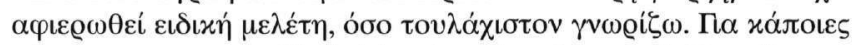

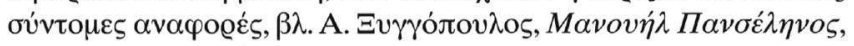

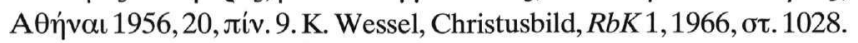

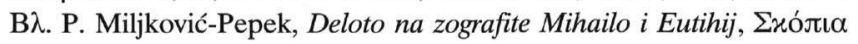

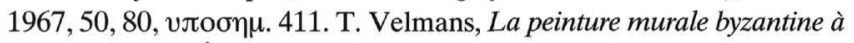

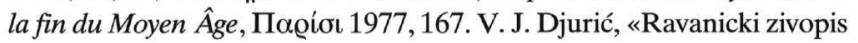

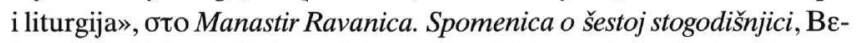

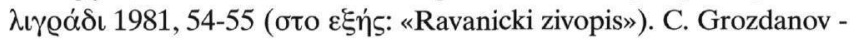

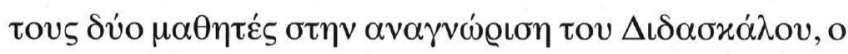

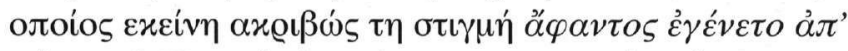

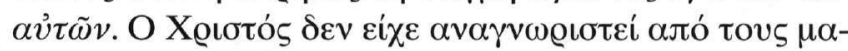

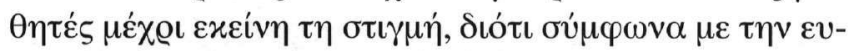

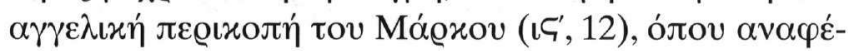

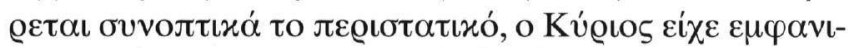

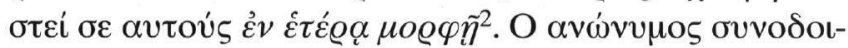

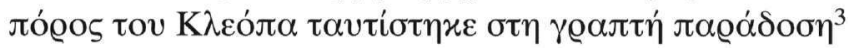

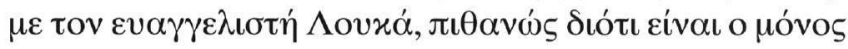

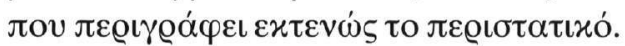

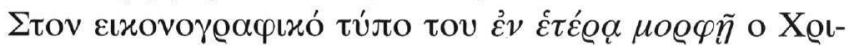

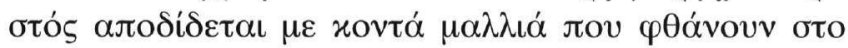

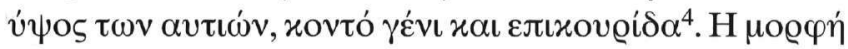

D. Ćornakov, «Istorijcki portreti ou Pološkom II», Zograf 15 (1984),

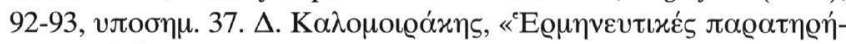

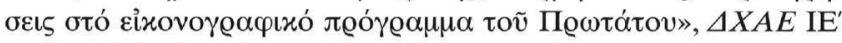

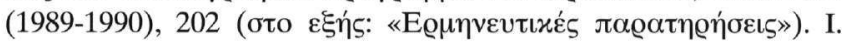
Spatharakis, "An Exceptional Representation of the Supper at Emmaus in the Church of St. Antonios at Vrontisi, Crete», Studies in

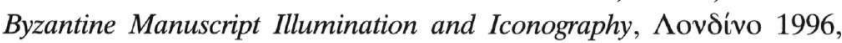

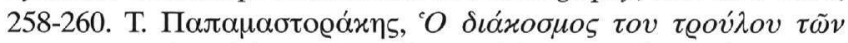

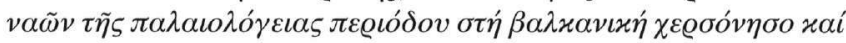

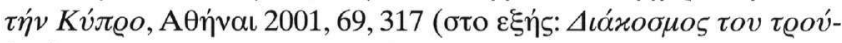
$\lambda o v)$. B. Todic, Serbian Medieval Painting. The Age of King Milutin, BE-

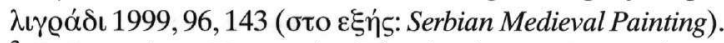

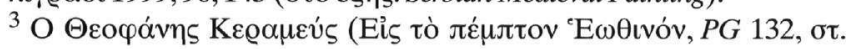

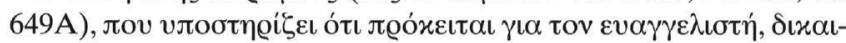

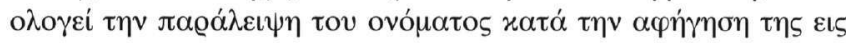

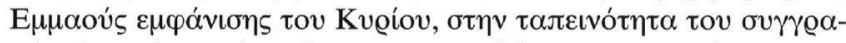

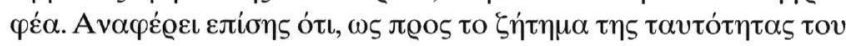

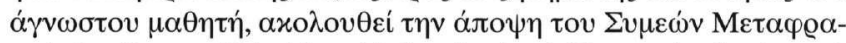

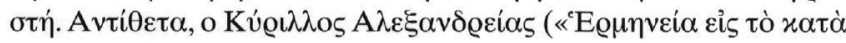

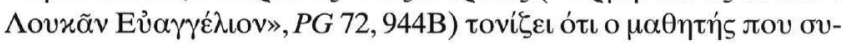

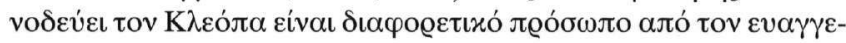

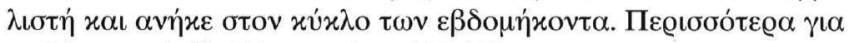

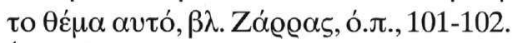

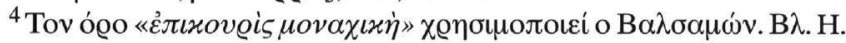

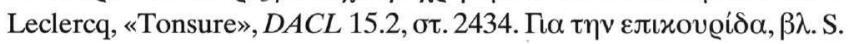
Radojčić, «Tonzure Sv. Save», Godisnjak Muzeja Juzne Srbije, I, ¿xó-

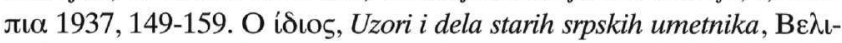

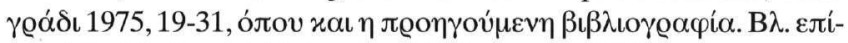

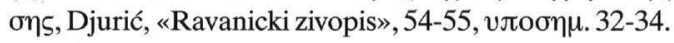




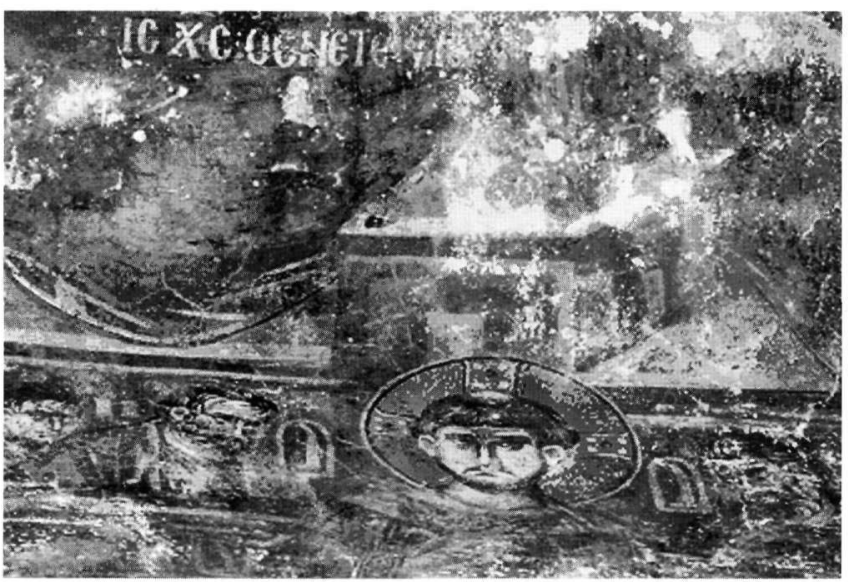

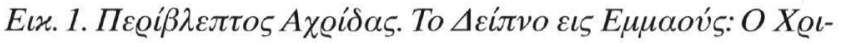

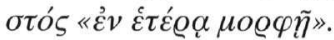

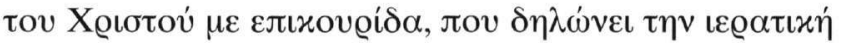

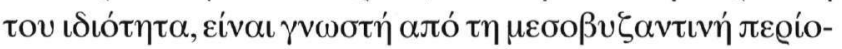

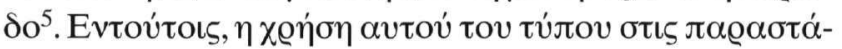

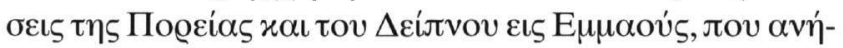

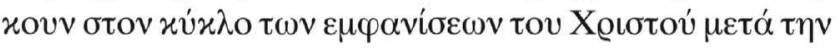

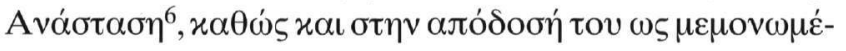

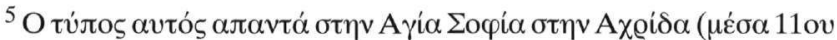

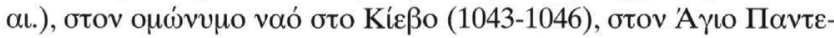

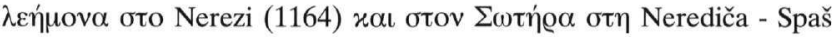

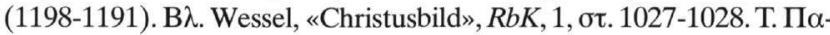

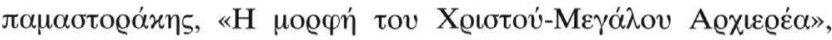

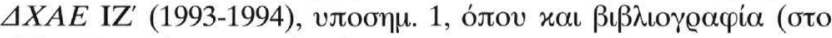

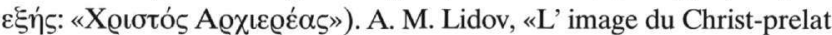
dans le programme iconographique de Sainte-Sophie d'Ohride», Arte Cristiana 79 (1991), 245-250. O í́ı૬, «Hristos-sviashennik' v ikonograficheskih programmah XI-XII vekov», VizVrem 55 (1994), 187-193.

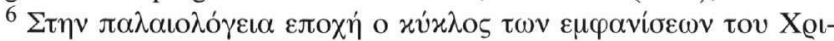

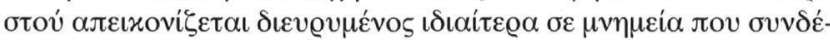

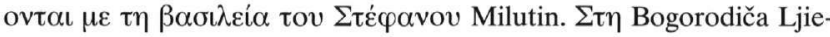

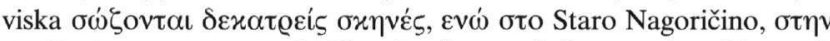
Gračanica, $x \alpha \iota \sigma \tau \eta \mu$ ov

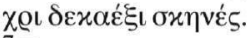

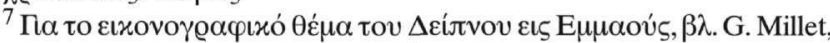
Recherches sur l' iconographie de l'Évangile aux XIVe, XV et XVIe siècles

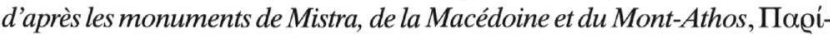

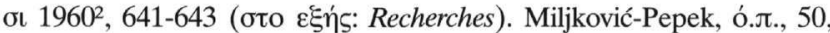
$\sigma \chi \varepsilon \dot{\delta}$. III. 14. K. Wessel, «Die Erscheinungen des Auferstandenen», RbK 2, oт. 386-387. G. Schiller, Ikonographie der christlichen Kunst, 3, Gütersloh 1971, 101-103. Spatharakis, ó.л., 249-262. Todić, Serbian

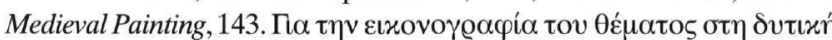

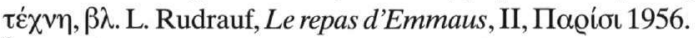

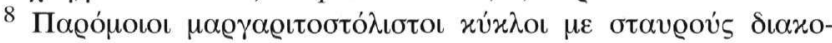

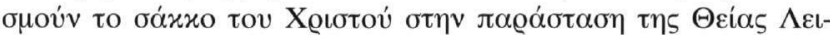

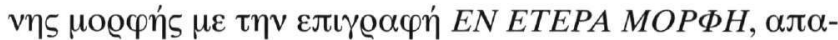

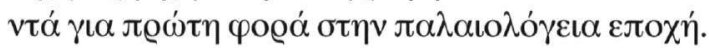

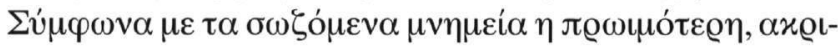

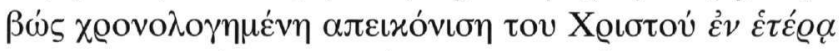

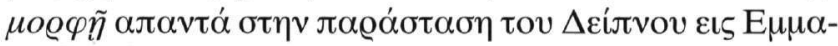

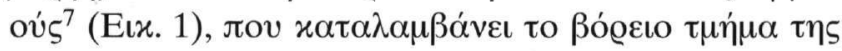

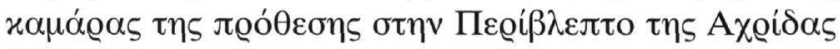

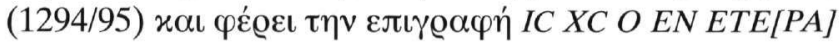

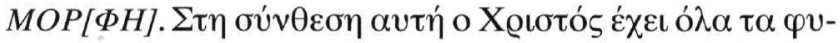

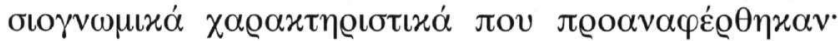

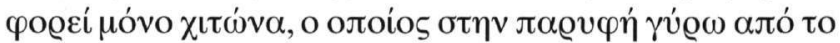

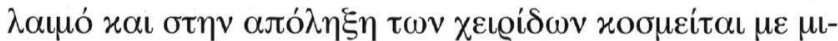

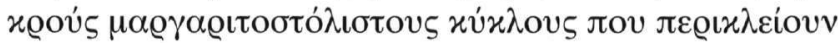

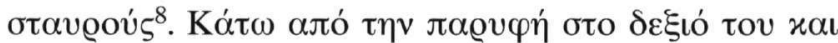

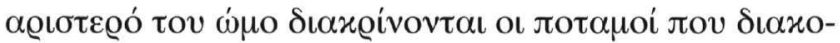

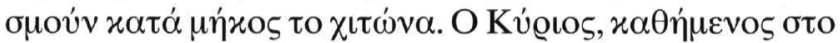

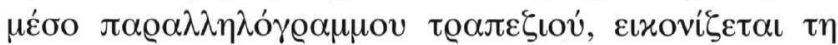

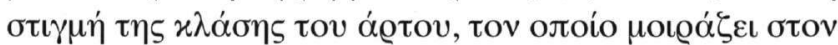

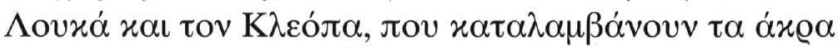

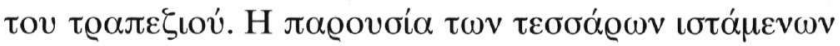

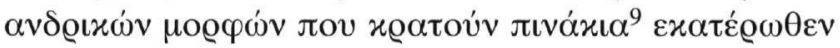

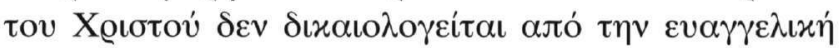

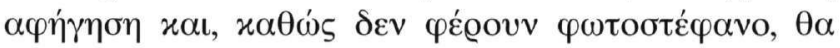
$\mu \pi$

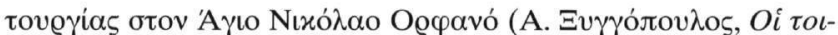

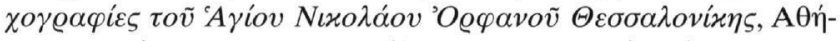

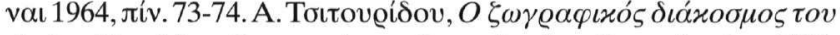

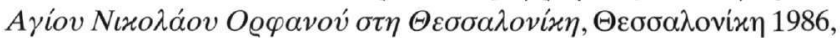

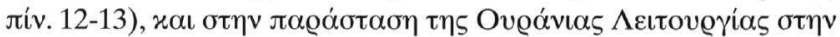

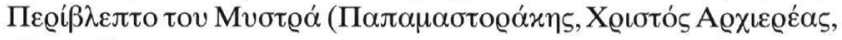
$68, \varepsilon(x .2)$.

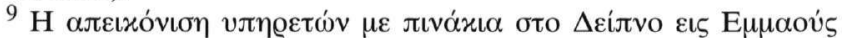

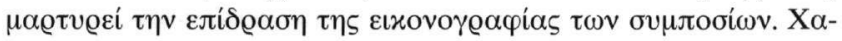

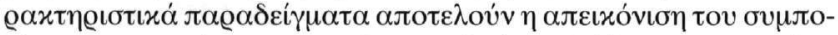

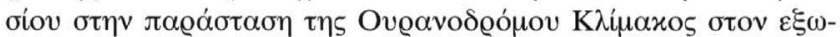

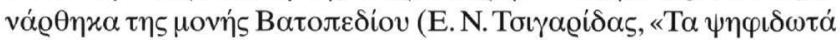

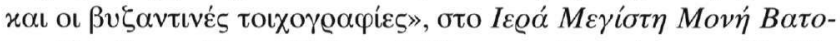

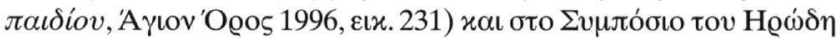

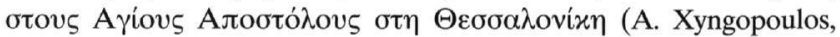
«Les fresques de l'église des Saints Apôtres à Thessalonique», oto Art et Société à Byzance sous les Paléologues. Actes du colloque organisé par l'Association internationale des Études Byzantines à Venise en septembre 1968, Bevetía 1971, عıx. 10. C. Stephan, Ein byzantinisches Bildensemple. Die Mosaiken und Fresken der Apostelkirche zu Thessaloniki, Baden-

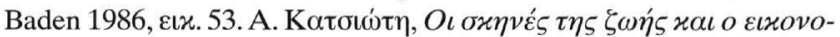

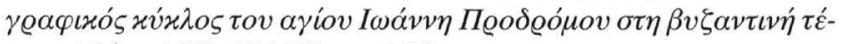

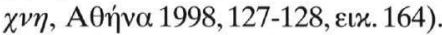

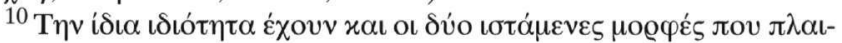

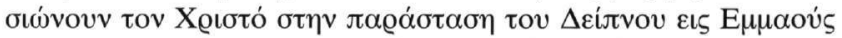

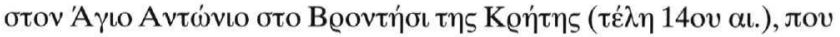

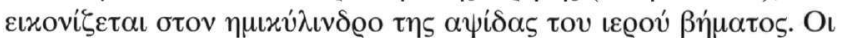




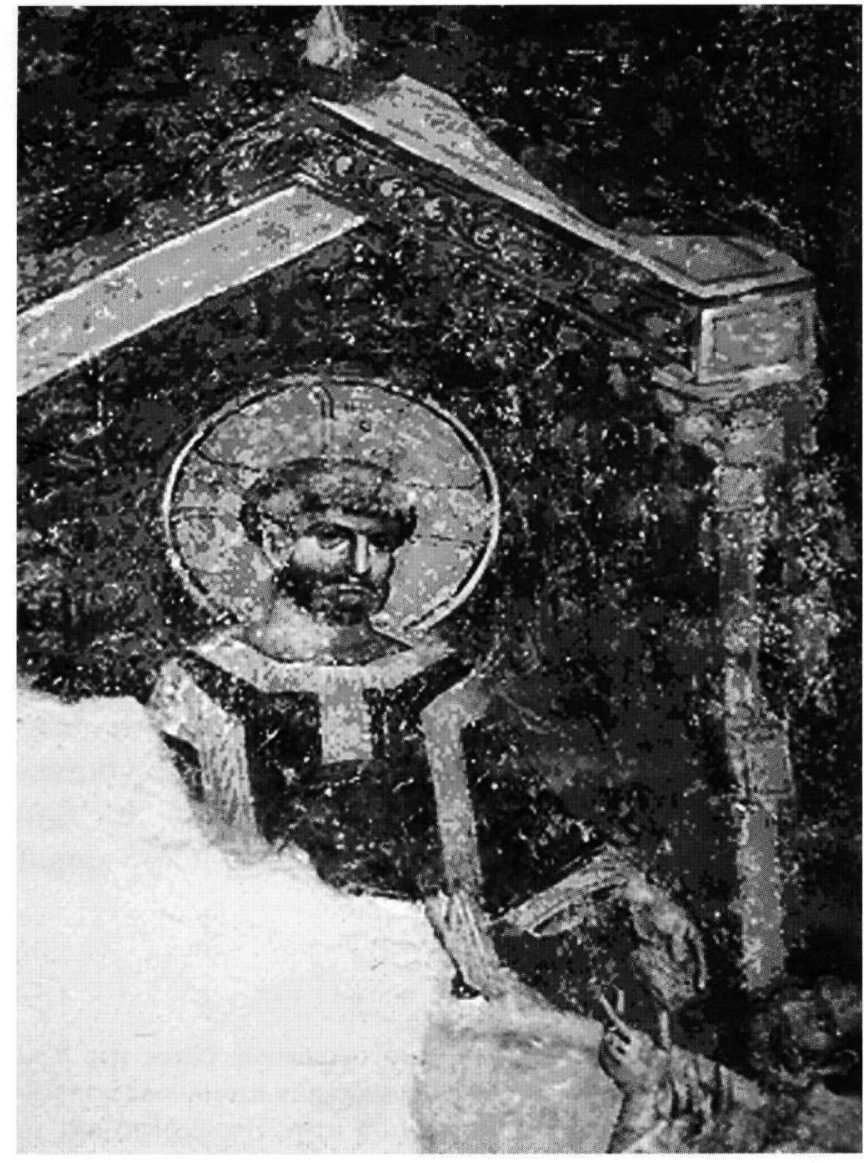

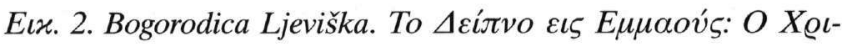

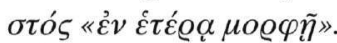

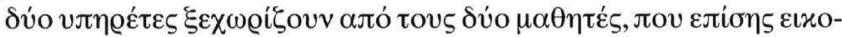

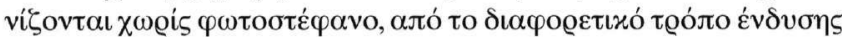

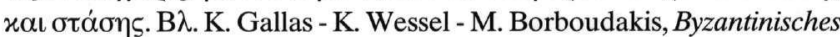

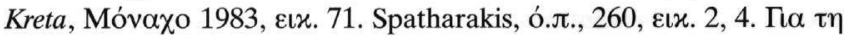

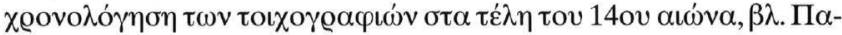

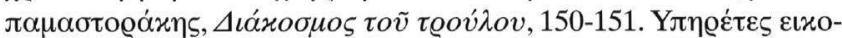

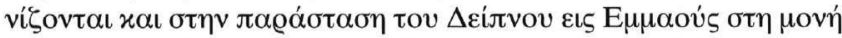

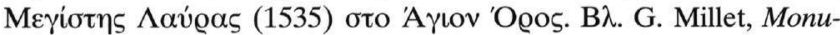

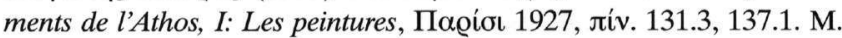
Chatzidakis, «Recherches sur le peintre Théophane le Cretois», DOP 23-24 (1969-1970), 331-332, عเx. 105.

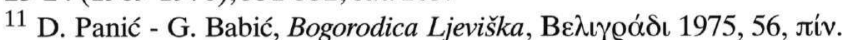

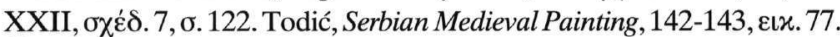

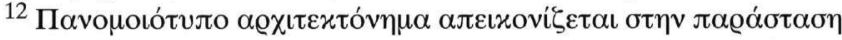

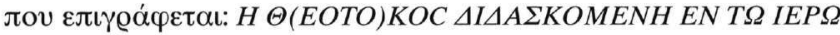

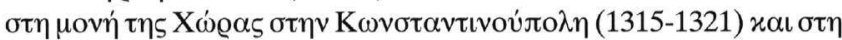

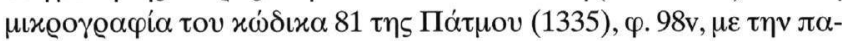

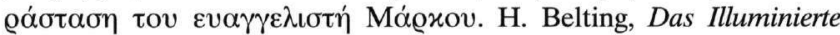

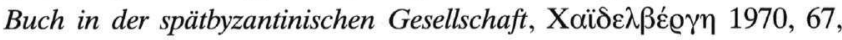

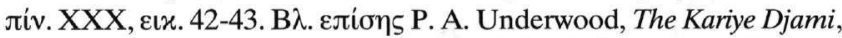

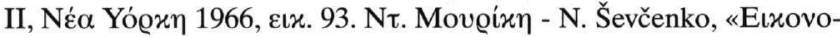

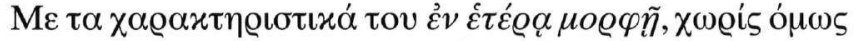

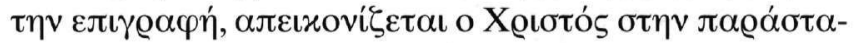

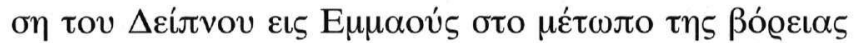

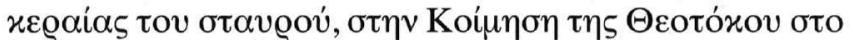
Prizren (Bogorodica Ljeviška) (1309-1313) ${ }^{11}$. Kó $\omega \omega \alpha \pi$ ó

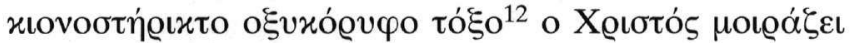

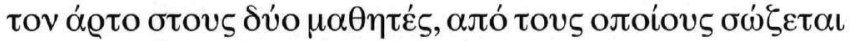

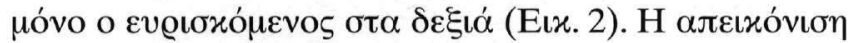

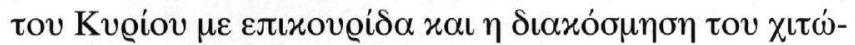

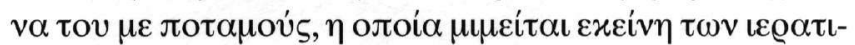

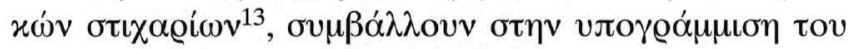

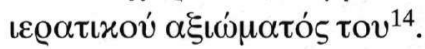

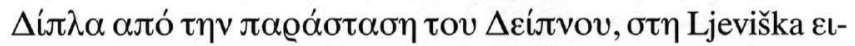

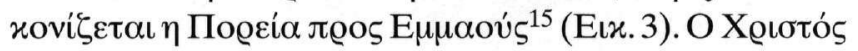

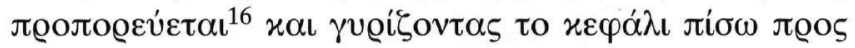

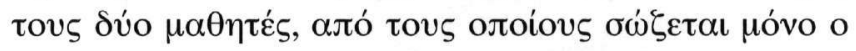

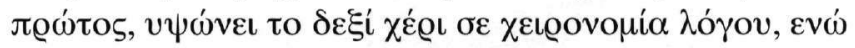

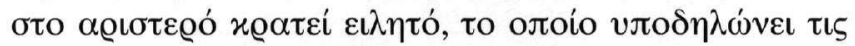

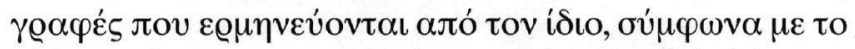

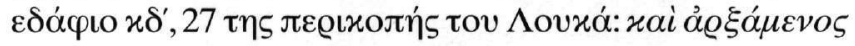

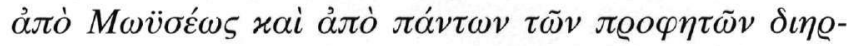

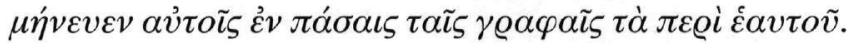

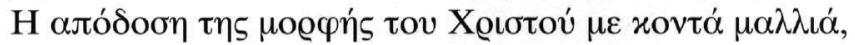

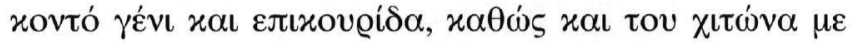

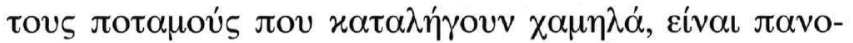

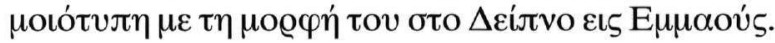

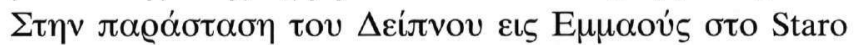

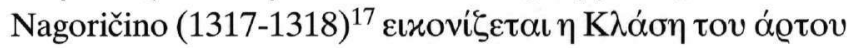

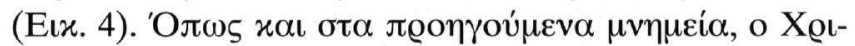

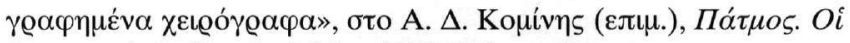

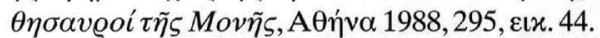

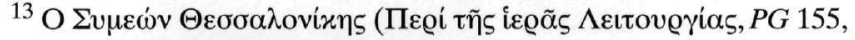

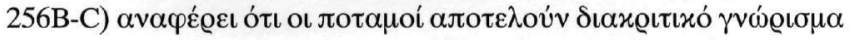

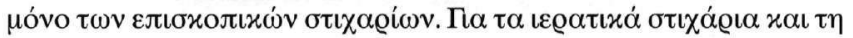

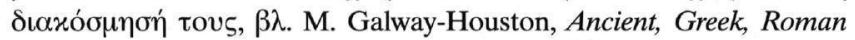

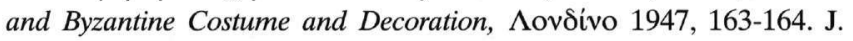
Braun, Die liturgische Gewandung im Occident und Orient, Darmstadt

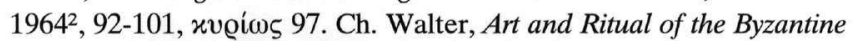

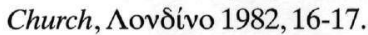

${ }^{14}$ Djurić, «Ravanicki zivopis», 55. Todić, Serbian Medieval Painting, 143. ${ }^{15}$ S. Radojčić, «Die Enstehung der Malerei der paläologischen Renais-

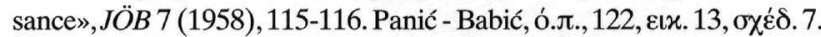

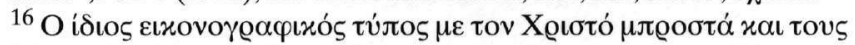

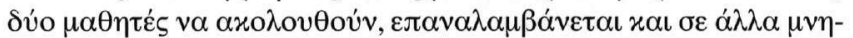

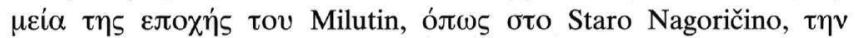

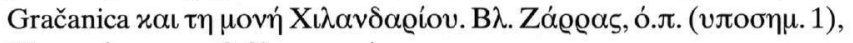

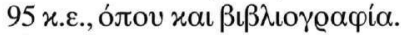

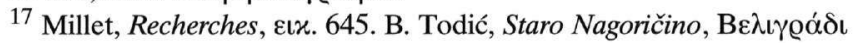

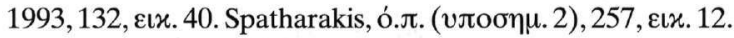




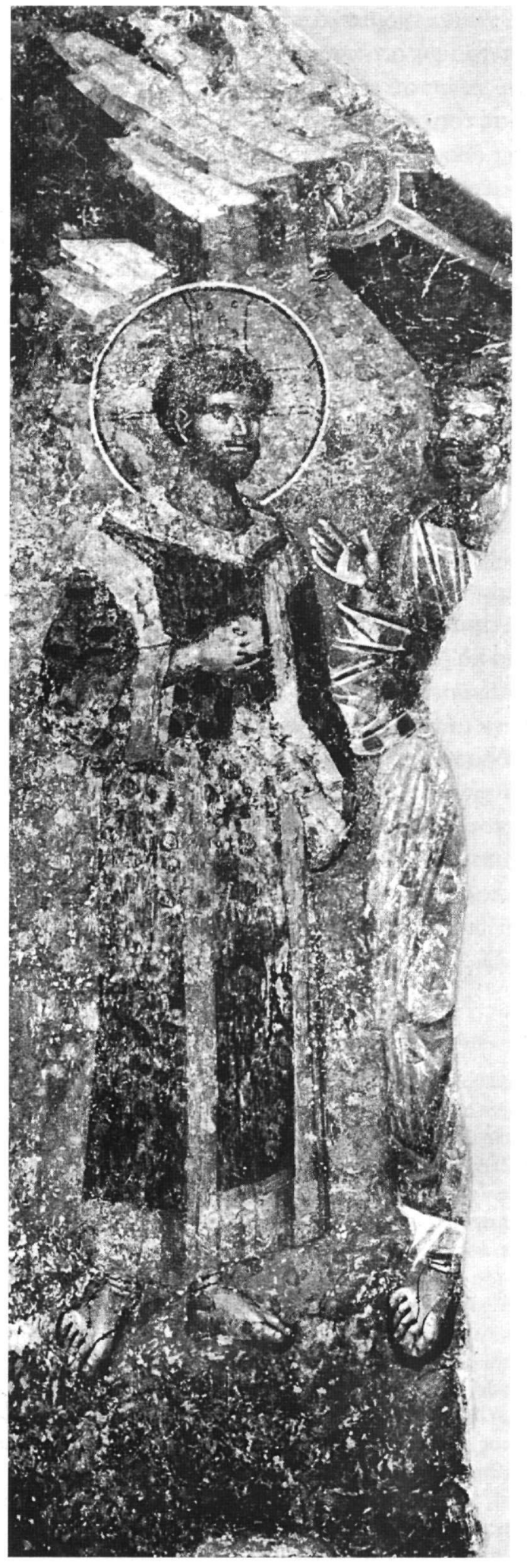

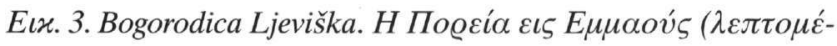
$\varrho \varepsilon \iota \alpha)$.

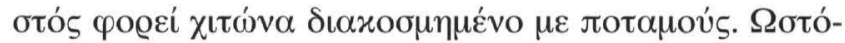

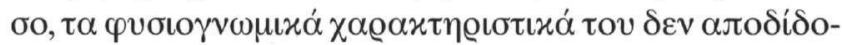

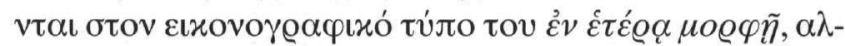

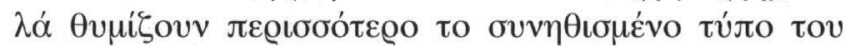

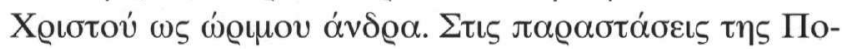

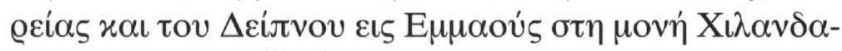

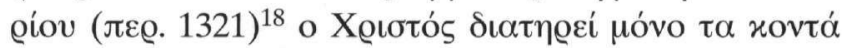

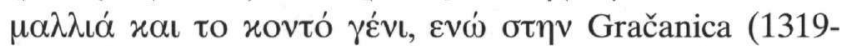

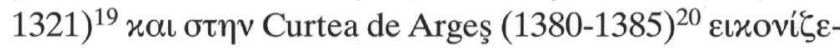

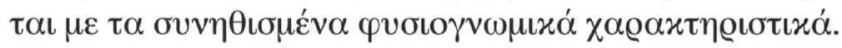

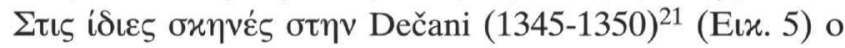

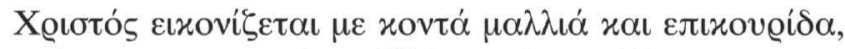

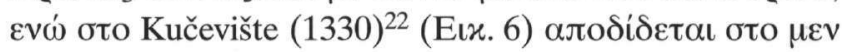

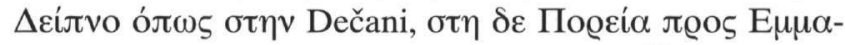

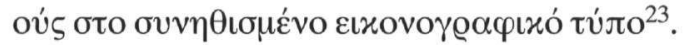

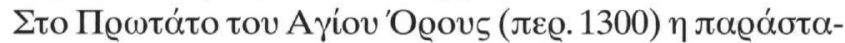

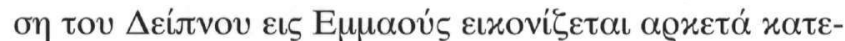

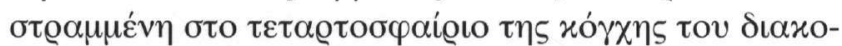

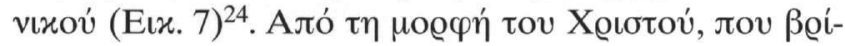

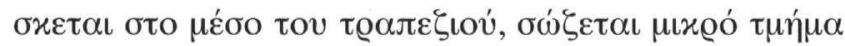

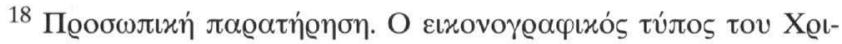

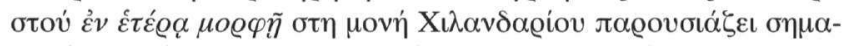

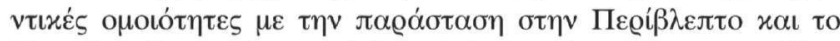

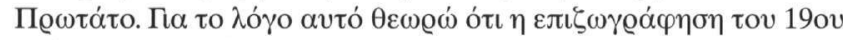

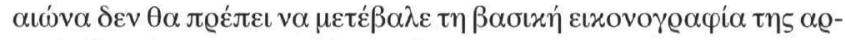

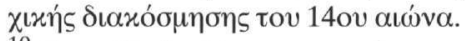

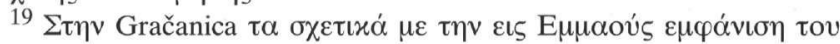

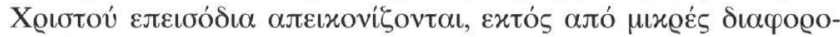

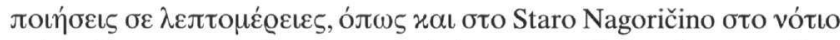

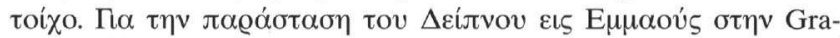

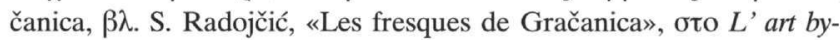

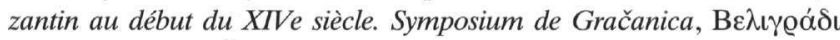
1978, عıx. 3. B. Živković, Gračanica. Les dessins des fresques (Les

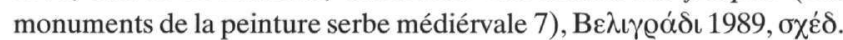

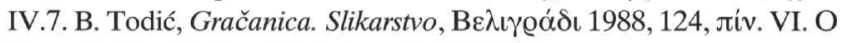

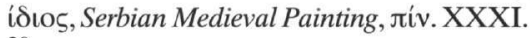

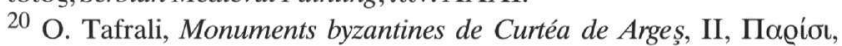
1931, лív. XXVIII.1. M. Musicescu - G. Ionescu, Biserica domnească

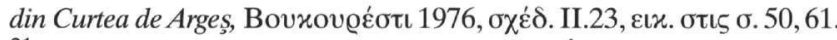

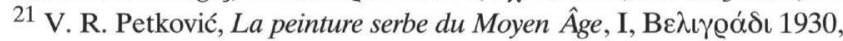

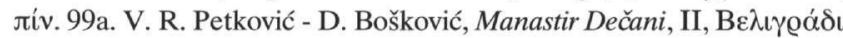
1941, jív. CCXVIII. B. Popović, «Program zivopisa ou Oltarskom

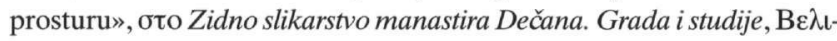

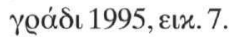

${ }^{22}$ I. Djordjević, «Slikarstvo XIV veka u crkvi sv. Spasa u selu Kučevištu», ZLU 17 (1981), 98, عเx. 24

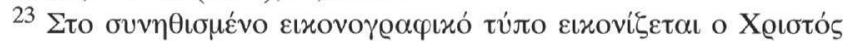

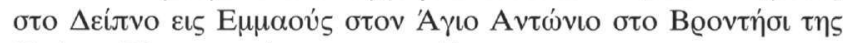

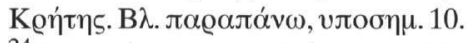

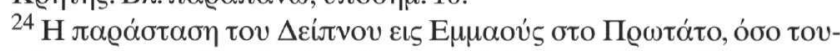

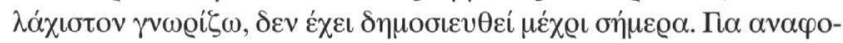




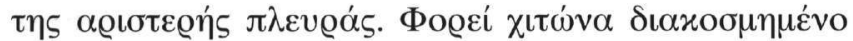

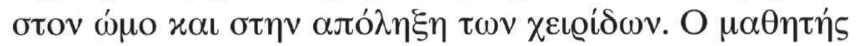

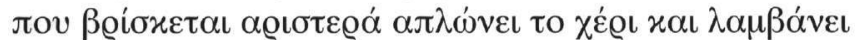

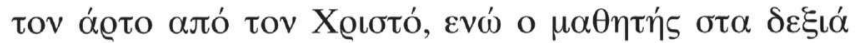

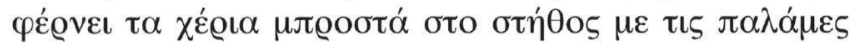

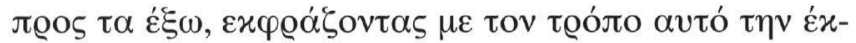

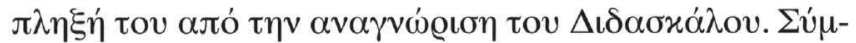

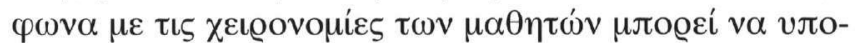

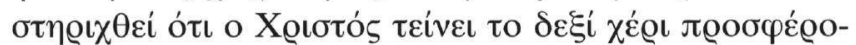

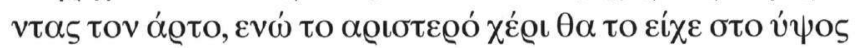

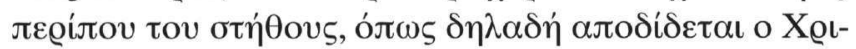

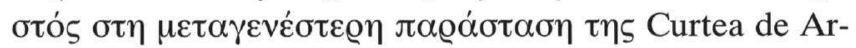

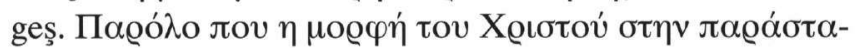

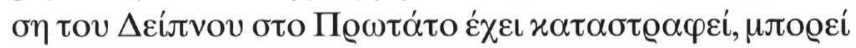

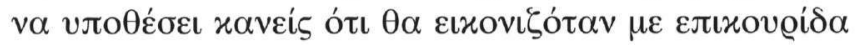

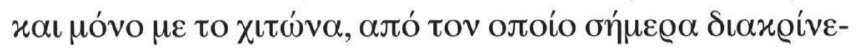

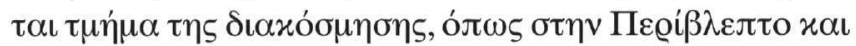
$\tau \eta v$ Bogorodica Ljeviška.

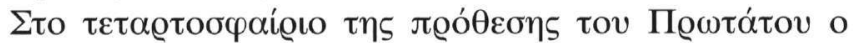

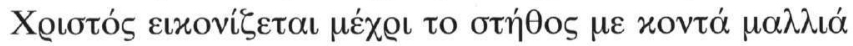

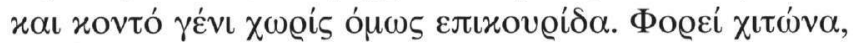

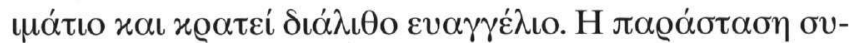

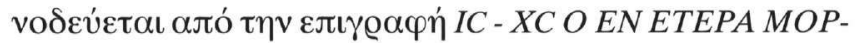

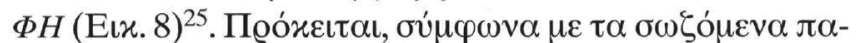

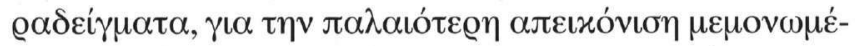

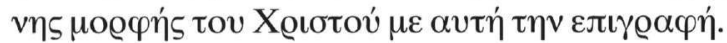

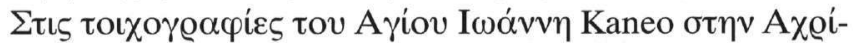

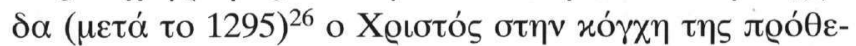

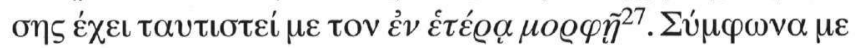

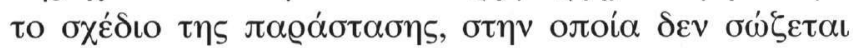

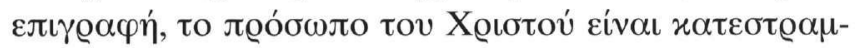

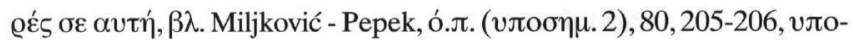

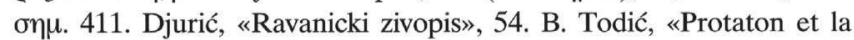

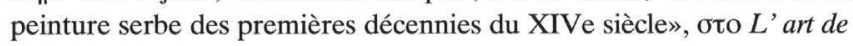
Thessalonique et de pays balkaniques et les courants spirituals au XIVe

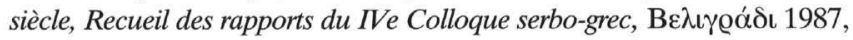
26. O ídıs, «Tradition et innovations dans le programme et l'ico-

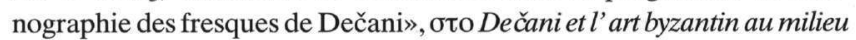
du XIVe siècle à l'occasion de la celebration de 650 ans du monastère de

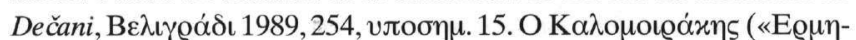

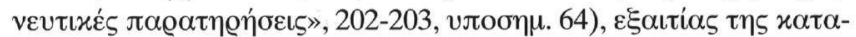

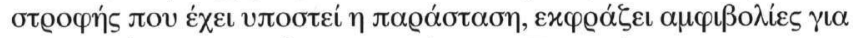

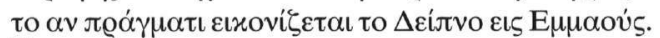

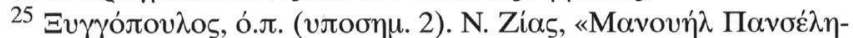

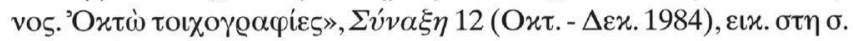

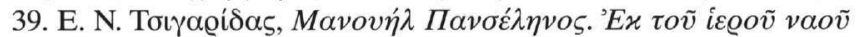

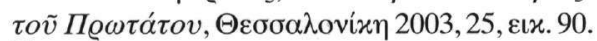

${ }^{26}$ O P. Miljković-Pepek («Contribution aux recherches sur l'evolution

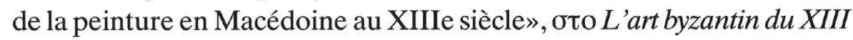

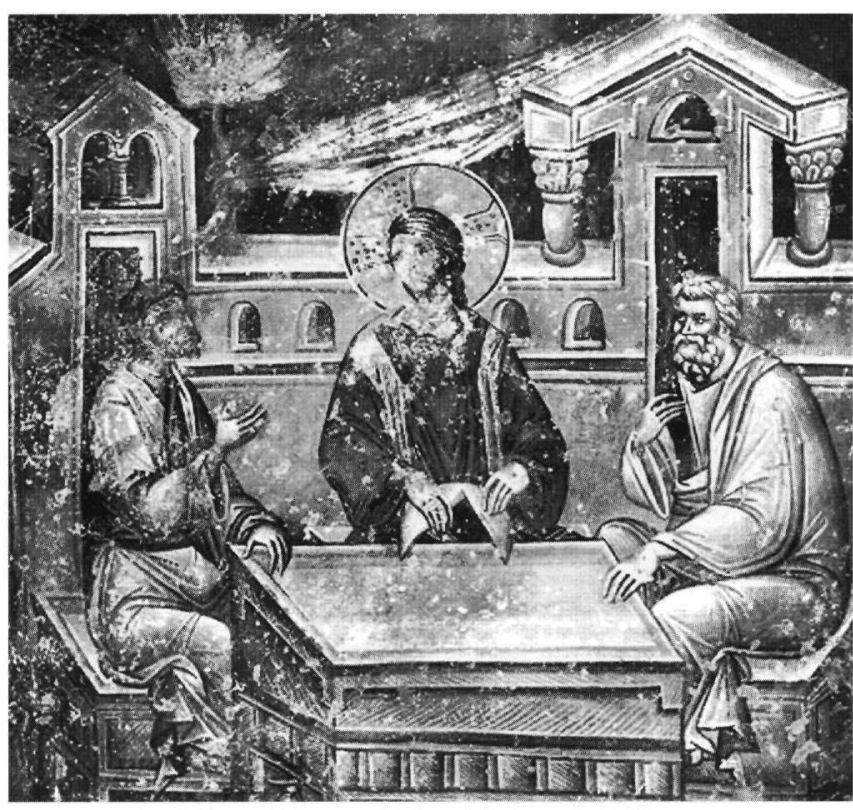

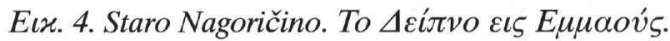

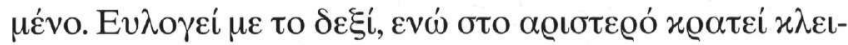

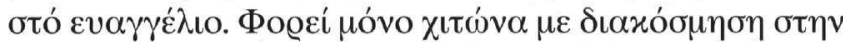

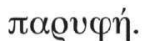

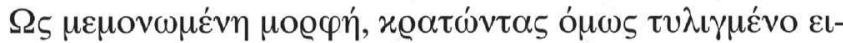

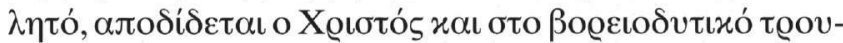

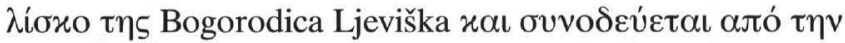

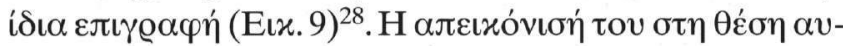

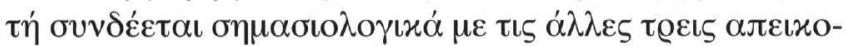

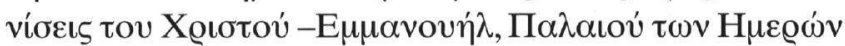

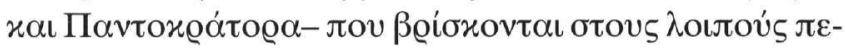

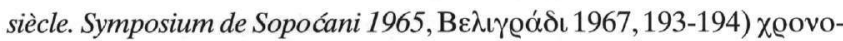

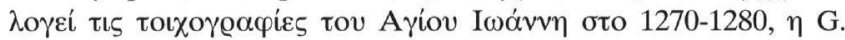
Babić («Peintures murales byzantines et de tradition byzantine (10811453). Possibilités et limites des analyses sociologiques», XVIIIe CIEB,

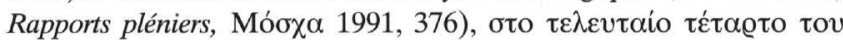

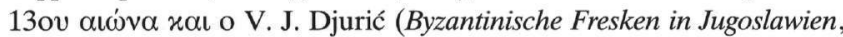

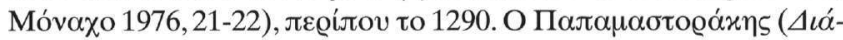

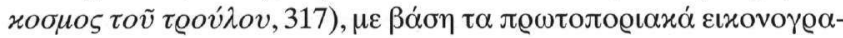

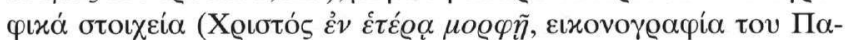

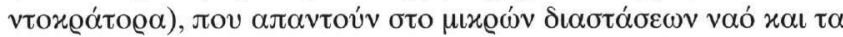

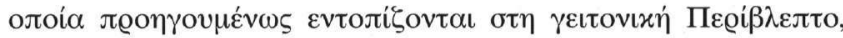

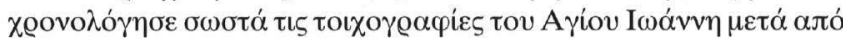

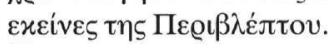

${ }^{27}$ P. Miljković-Pepek, «Crkvata sv. Jovan Bogoslov - Kaneo vo Ohrid»,

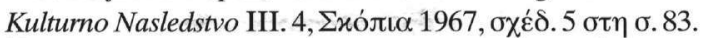

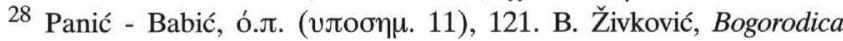
Ljeviška. Les dessins des fresques (Les monuments de la peinture serbe

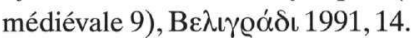




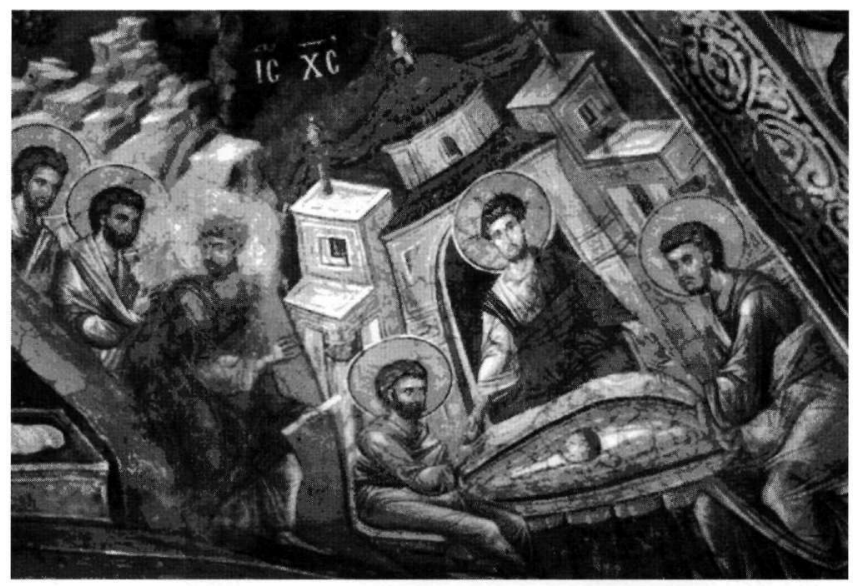

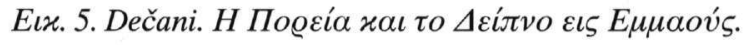

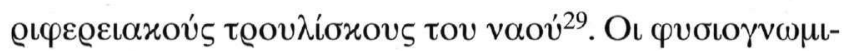

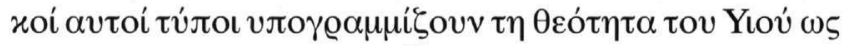

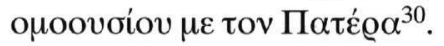

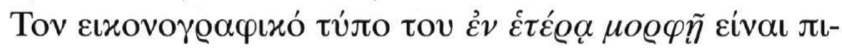

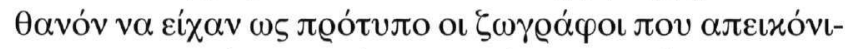

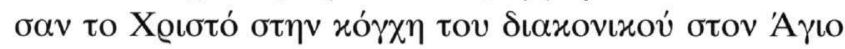

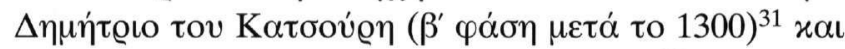

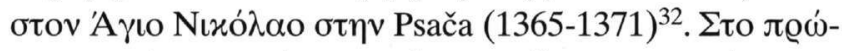

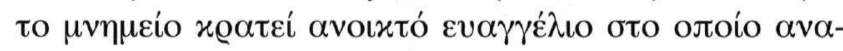

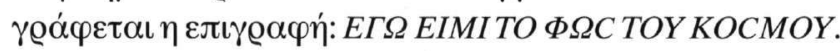

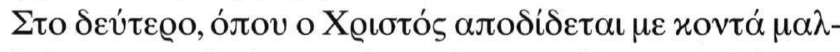

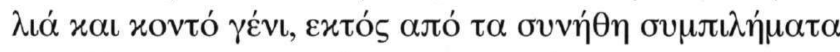

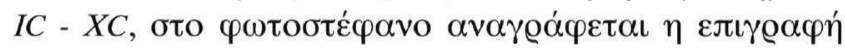

\footnotetext{
${ }^{29}$ Panić - Babić, ó.л., 120-121. Živković, ó.л., 12-14.

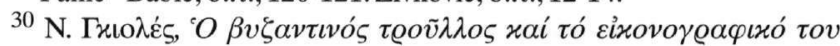

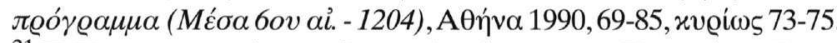

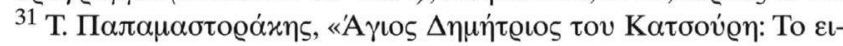

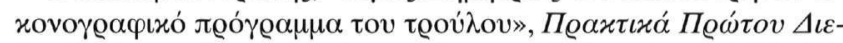

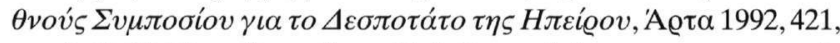

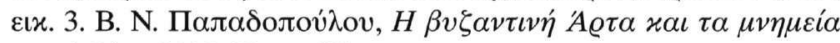
$\tau \eta \zeta$, A $\theta \dot{\eta} v \alpha$ 2002,32, عเx. 27.

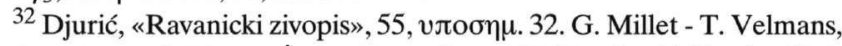

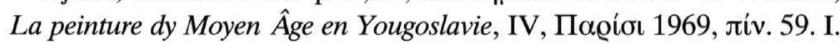

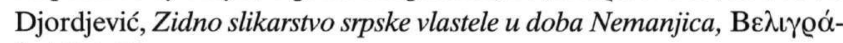
Sı 1994, 74 .

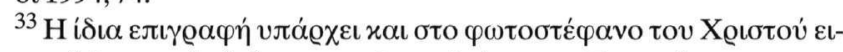

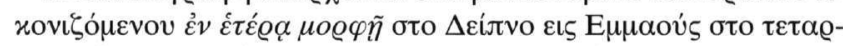

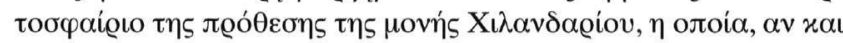

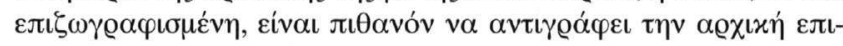

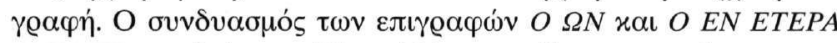

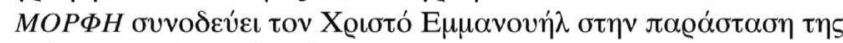

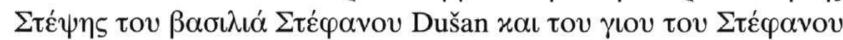

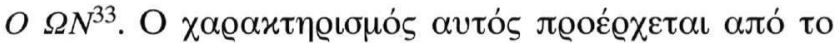

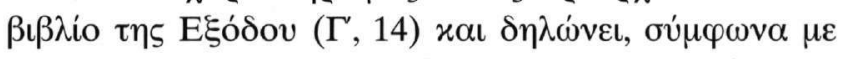

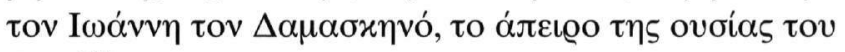
$\Theta \varepsilon o v^{34}$.

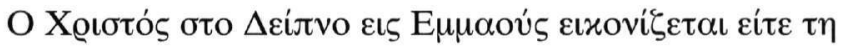

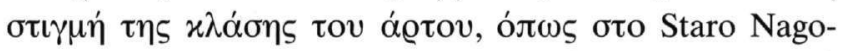

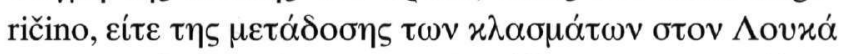

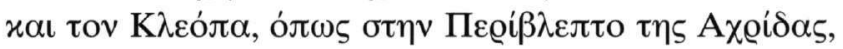

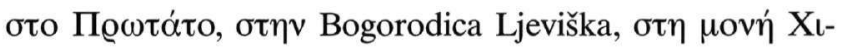

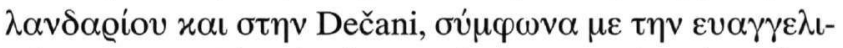

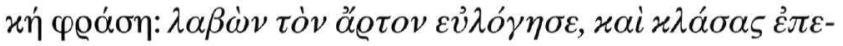

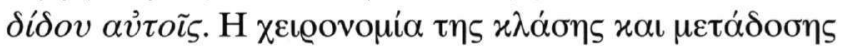

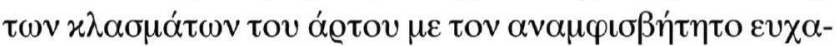

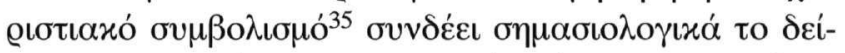

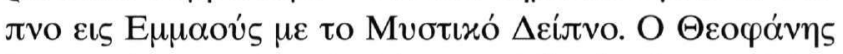

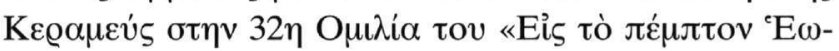

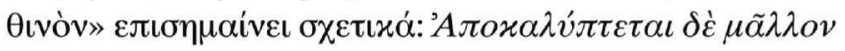

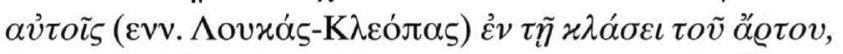
$\dot{\varepsilon} v \tau \tilde{\eta} \mu \varepsilon \tau \alpha \lambda \dot{\eta} \psi \varepsilon \iota \delta \eta \lambda \alpha \delta \dot{\eta} \tau \tilde{\omega} v \varphi \varrho \iota x \tau \tilde{\omega} v \mu v \sigma \tau \eta \varrho i ́ \omega v^{36}$.

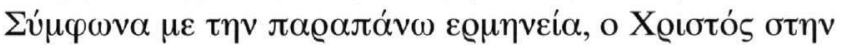

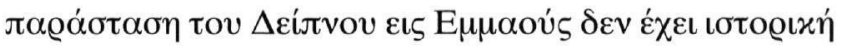

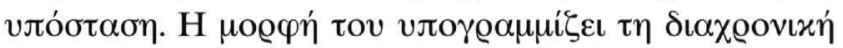

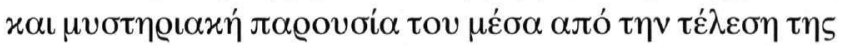

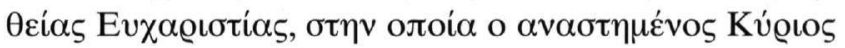

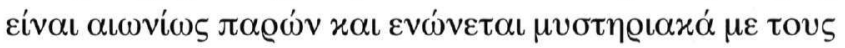
лıテтoús.

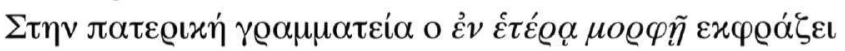

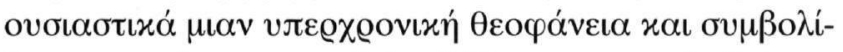

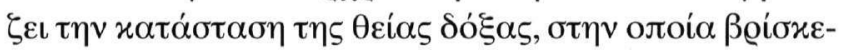

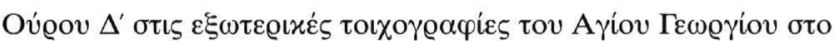

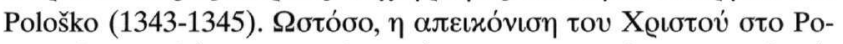

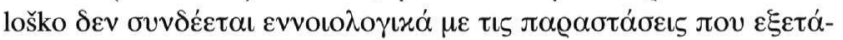

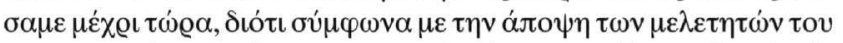

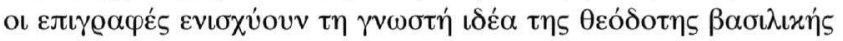

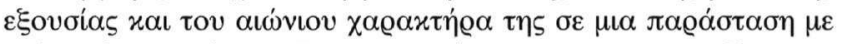

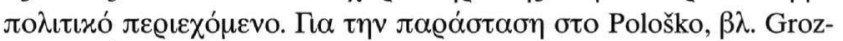

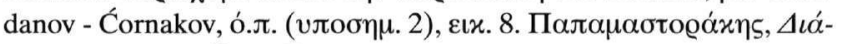

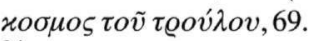

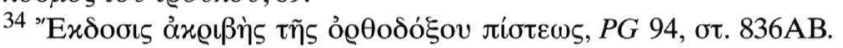

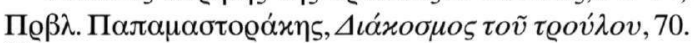

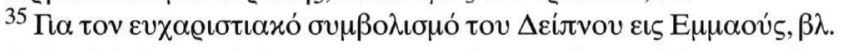
G. Bovini, La vita di Cristo nei mosaici di S. Apollinare di Ravenna, $\mathrm{P} \alpha-$ ßévva 1959, 95-97. H. Hallensleben, Die Malerschule des Königs Milutin, Giessen 1976, 47. Djurić, «Ravanicki zivopis», б. 54-55. Djor-

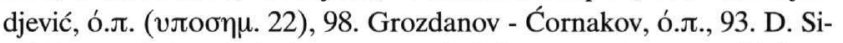
mić-Lazar, Kalenićet la dernière période de la peinture byzantine, $\Sigma$ xó $\iota \alpha$ 1995, 60-61.

${ }^{36} P G 132,657 \mathrm{C}$. 


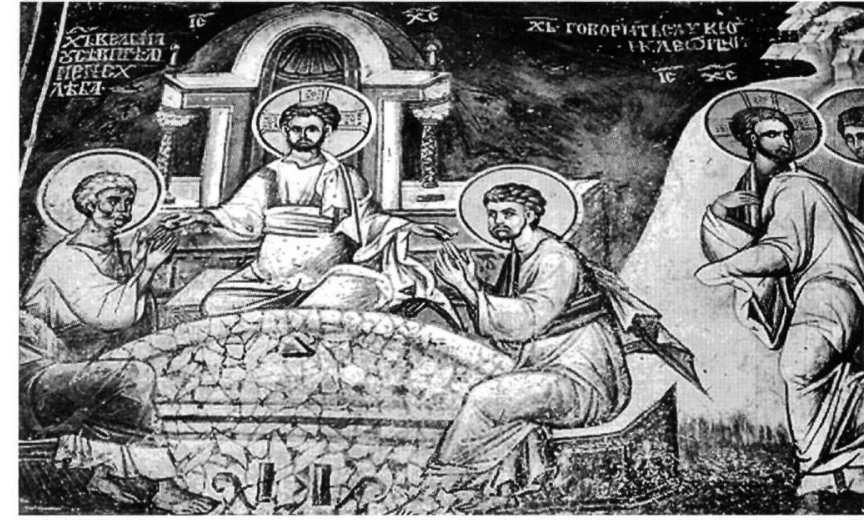

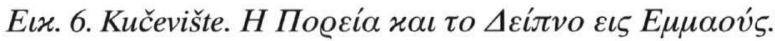

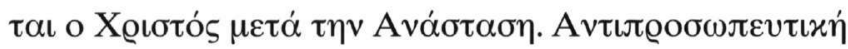

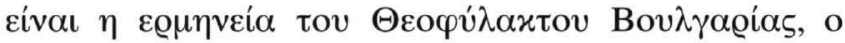

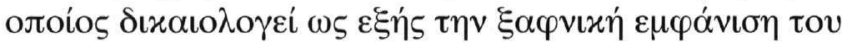

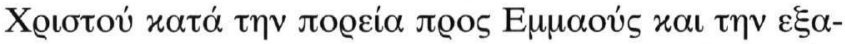

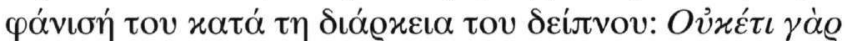

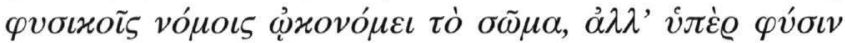

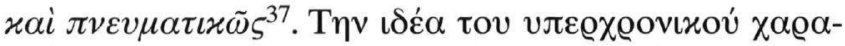

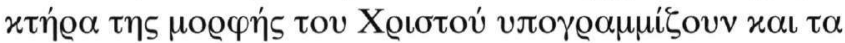

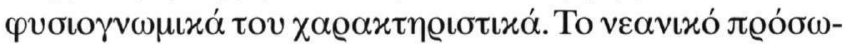

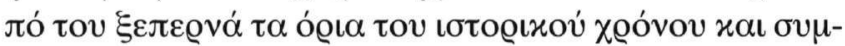

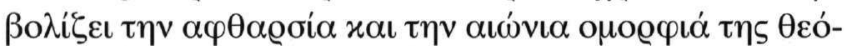

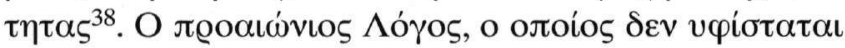

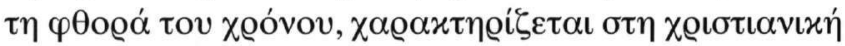

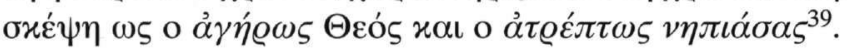

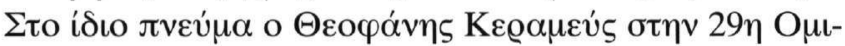

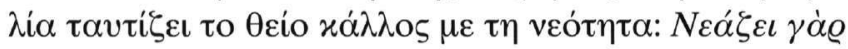

\footnotetext{
${ }^{37} P G 123, \sigma \tau .1113 \mathrm{~B}$.
}

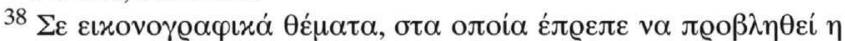

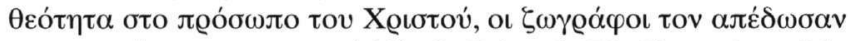
$\mu \varepsilon v \varepsilon \alpha v \iota x \alpha$ $\chi \alpha \varrho \alpha x \tau \eta \varrho \iota \tau \iota x \alpha \dot{\alpha} . \mathrm{B} \lambda$. G. Galavaris, The Illustrations of the

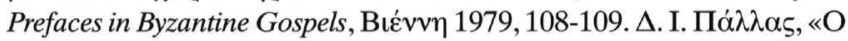

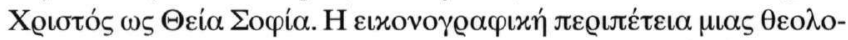

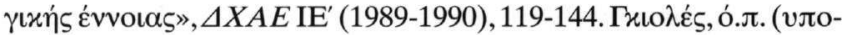

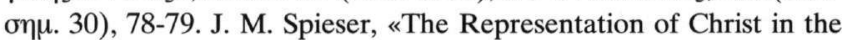
Apses of Early Christian Churches», Gesta 37/1 (1998), 69.

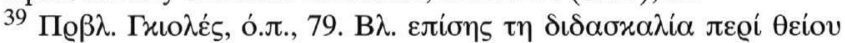

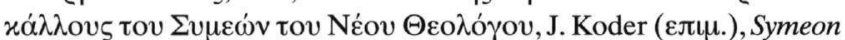

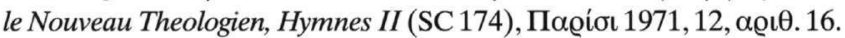
Galavaris, ó. $\pi ., 109$.

${ }^{40} P G 132,625 \mathrm{~A}$.

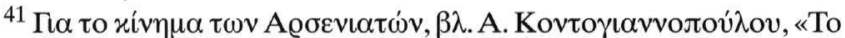

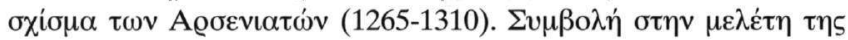

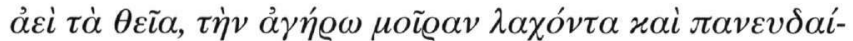
$\mu о v \alpha^{40}$.

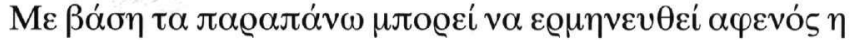

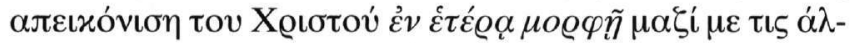

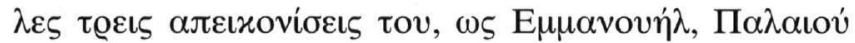

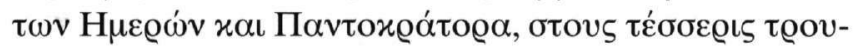

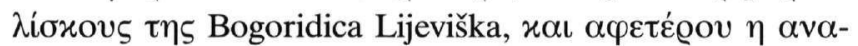

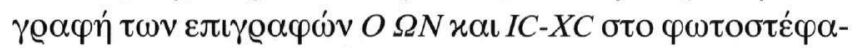

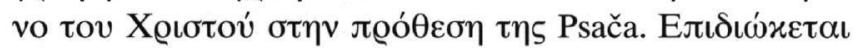

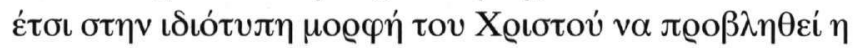

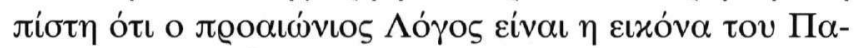

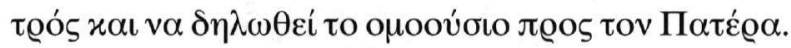

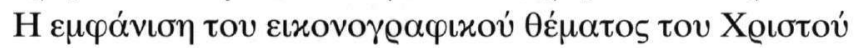

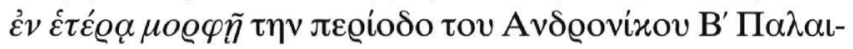

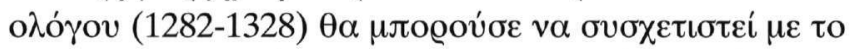

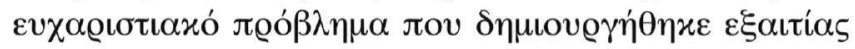

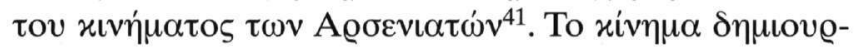

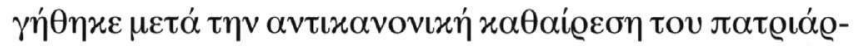

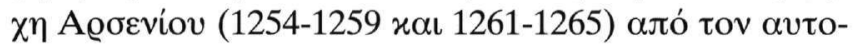

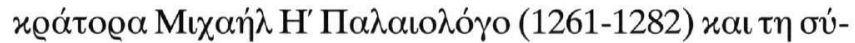

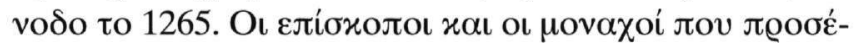

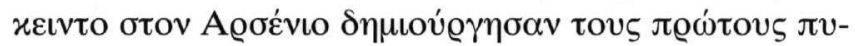

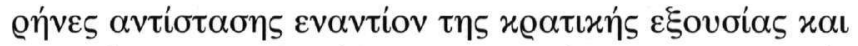

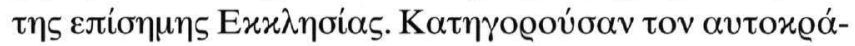

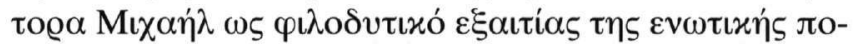

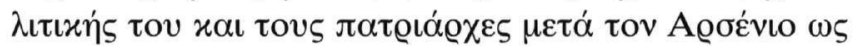

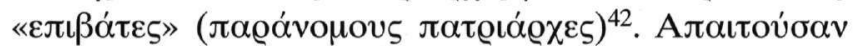

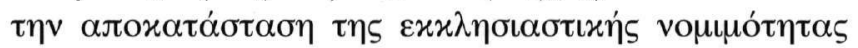

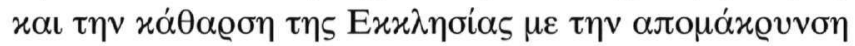

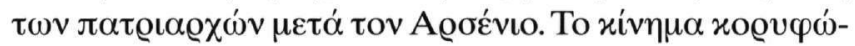

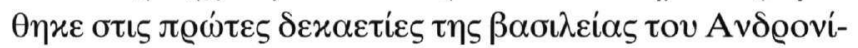

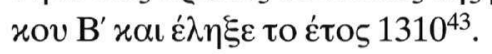

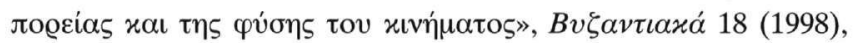

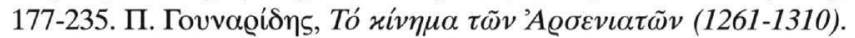

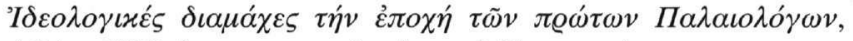

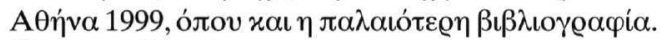

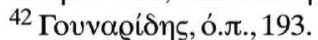

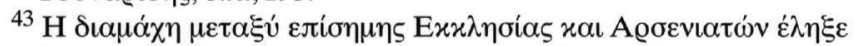

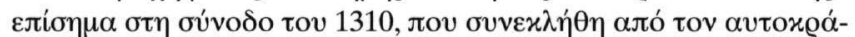

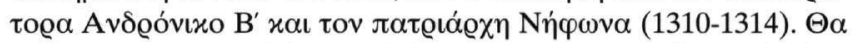

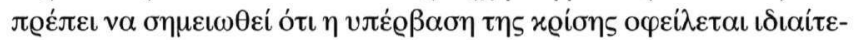

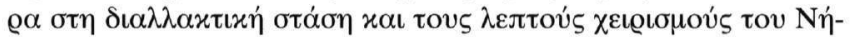

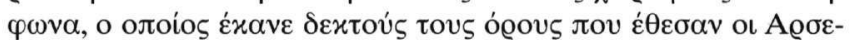

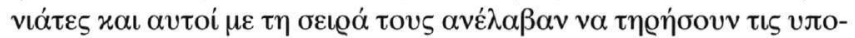

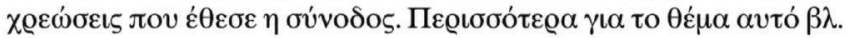
V. Laurent, «Les grandes crises religieuses à Byzance: La fin du schisme

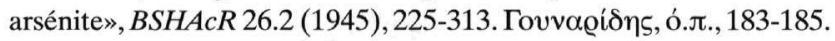




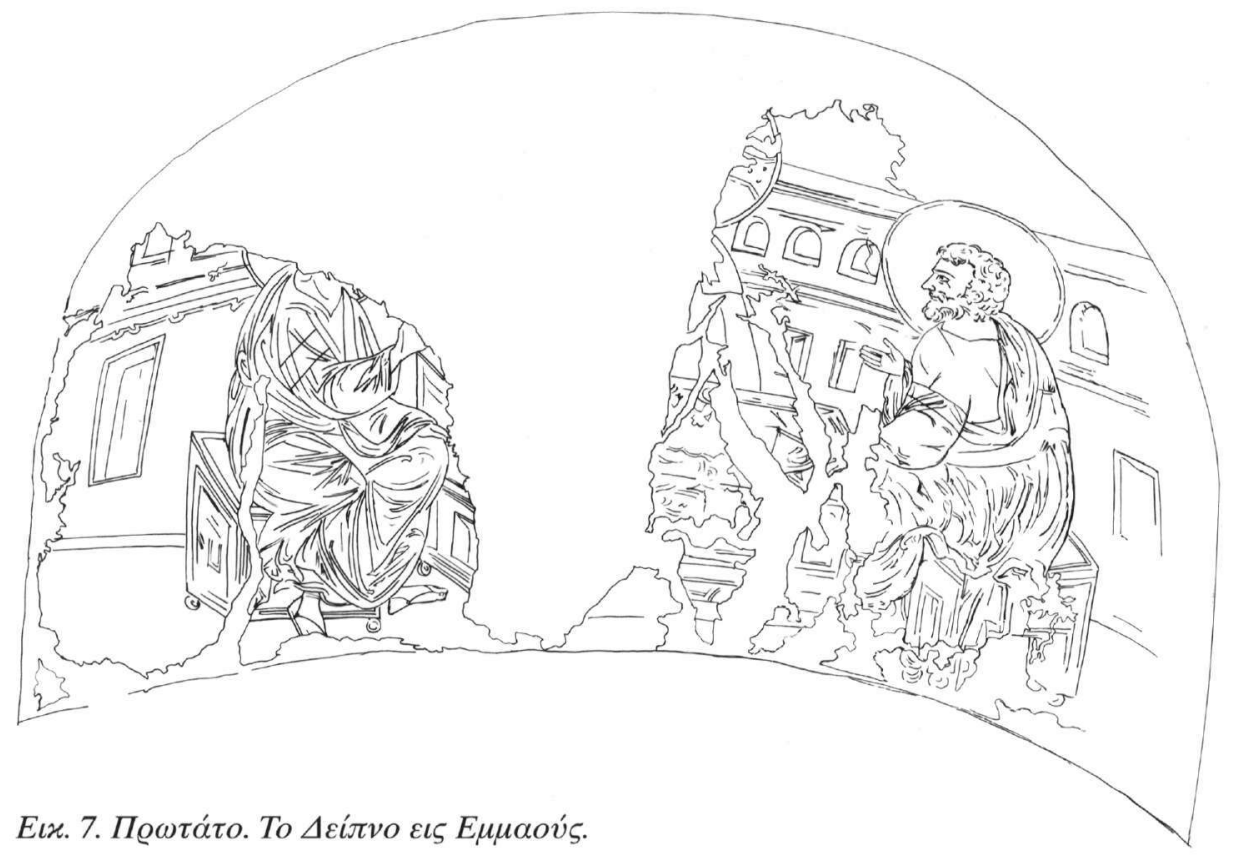

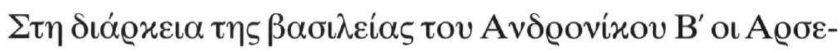

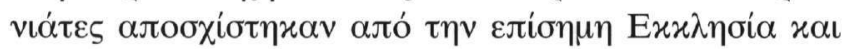

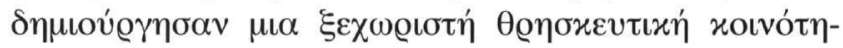

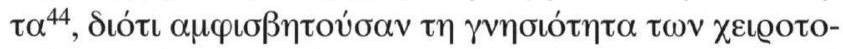

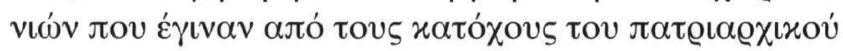

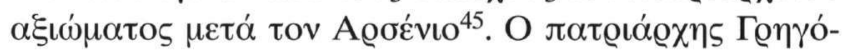

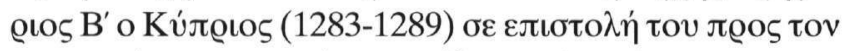

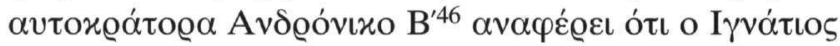

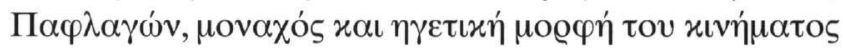

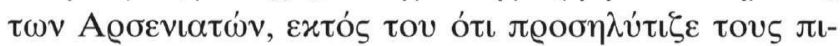

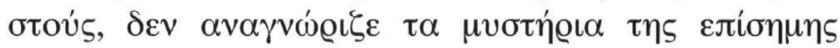

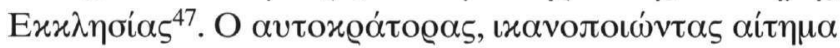

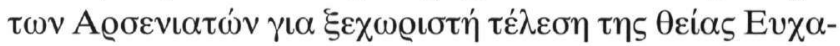

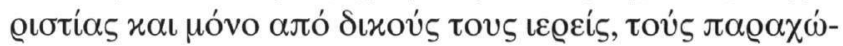

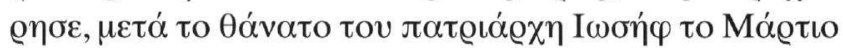

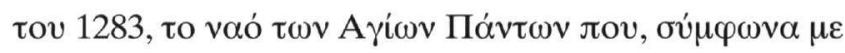

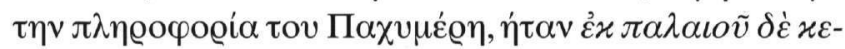

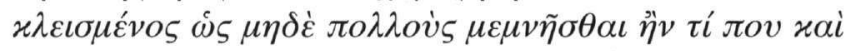

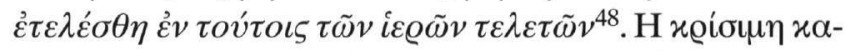

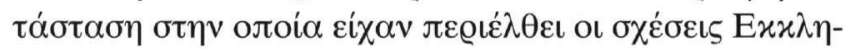

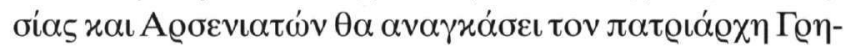

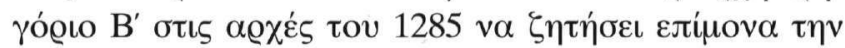

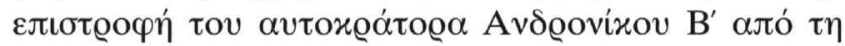

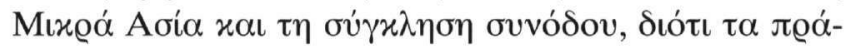

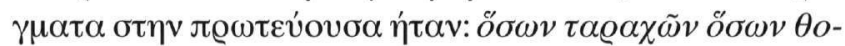

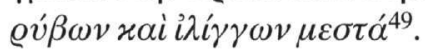

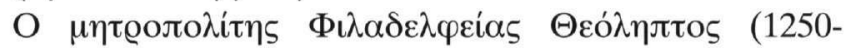

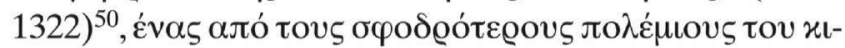

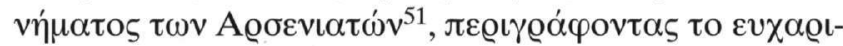

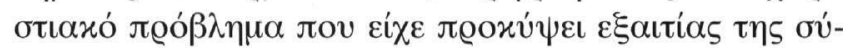

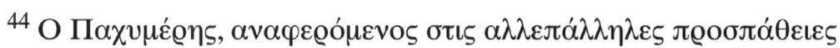

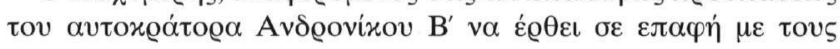

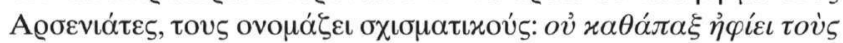

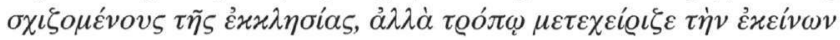

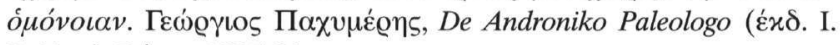
Bekker), Bóvvi 1835, 81.

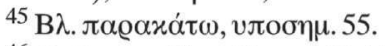

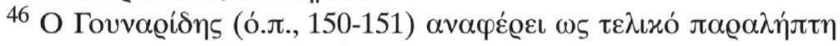

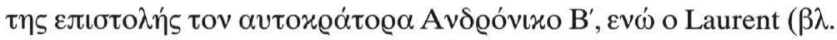

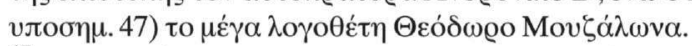

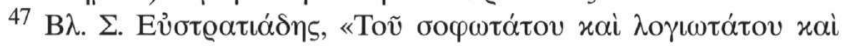

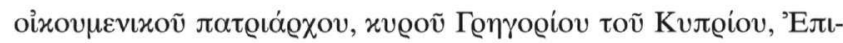

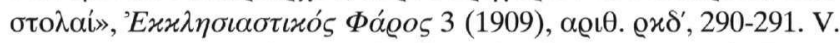
Laurent, Les regestes des actes du patriarchat de Constantinople, IV: Les

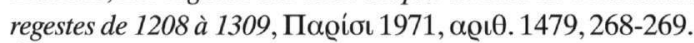

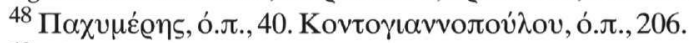

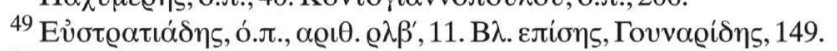

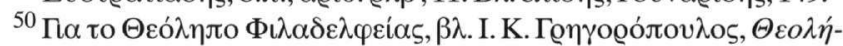
$\pi \tau o v$ Ф

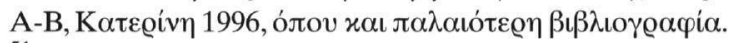

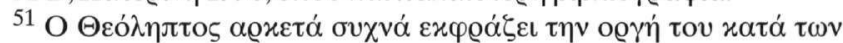

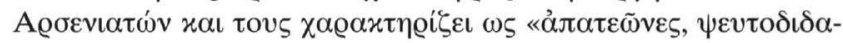




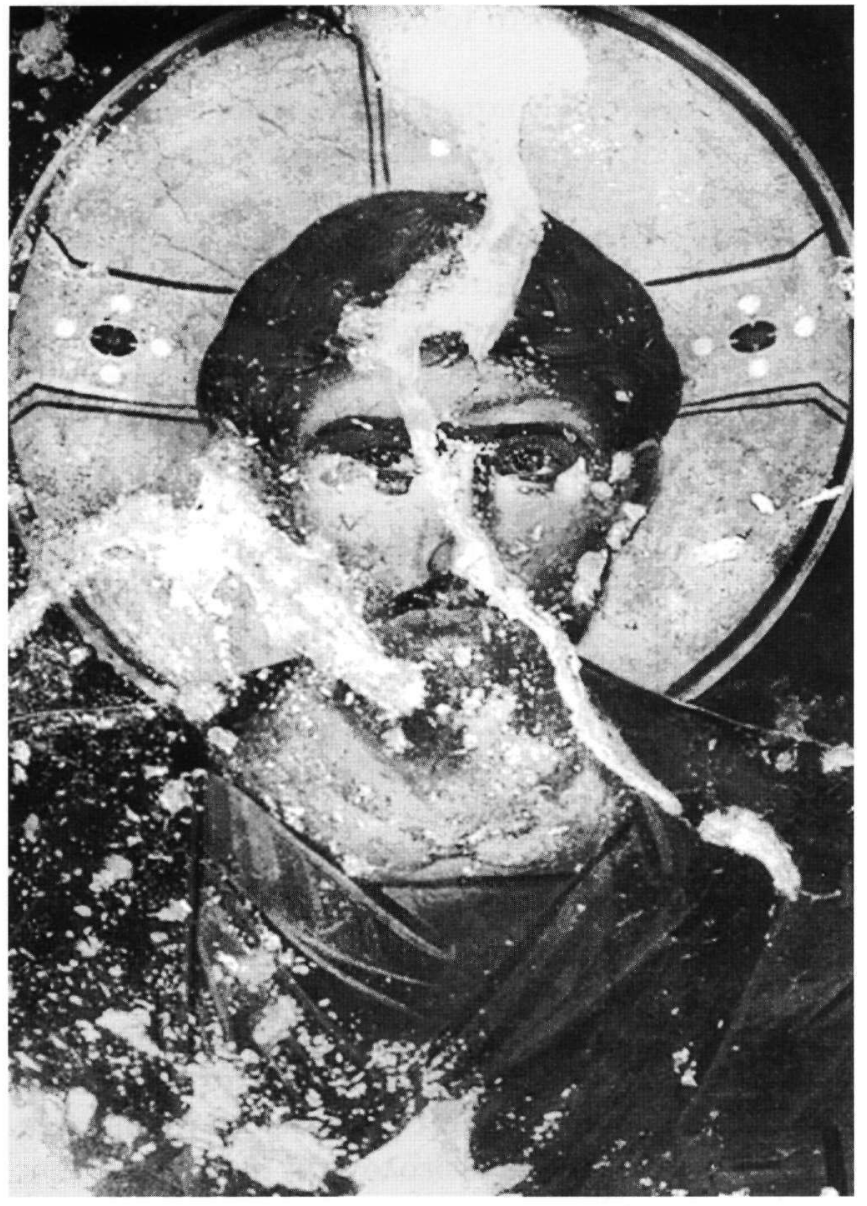

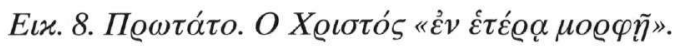

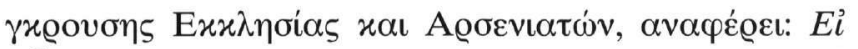

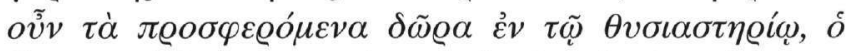

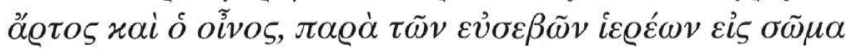

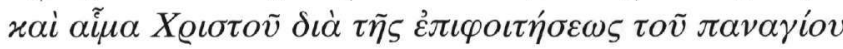

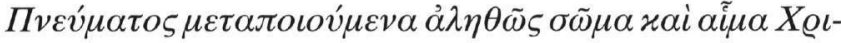

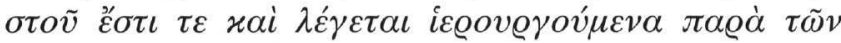

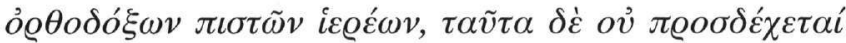

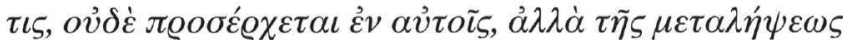

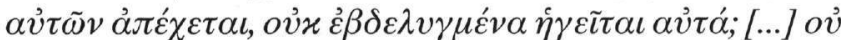

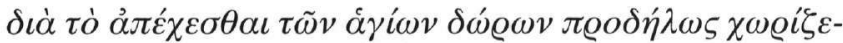

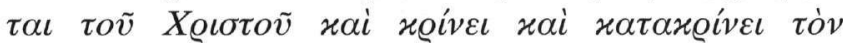

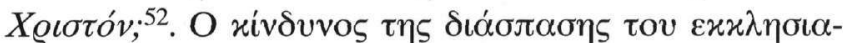

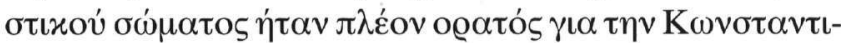

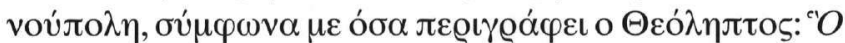

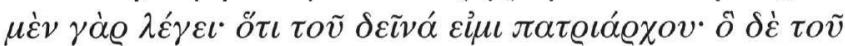

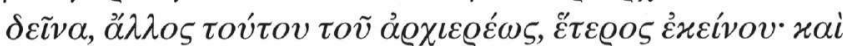

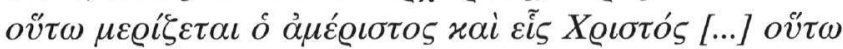

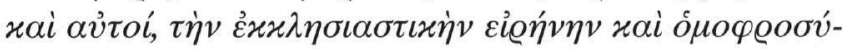

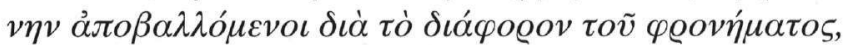

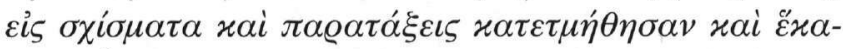
$\sigma \tau o \varsigma, \tilde{\omega} v \dot{\eta} \beta o v \lambda \dot{\eta} \theta \eta \tau \tilde{\omega} v \dot{\alpha} v \theta \varrho \omega \dot{\pi} \pi \omega v, \varepsilon i \zeta$ $\chi \varepsilon \varphi \alpha \lambda \dot{\eta} v \dot{\varepsilon} \alpha v \tau \tilde{\omega}$

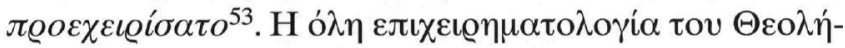

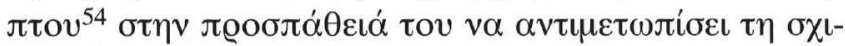

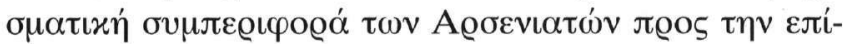

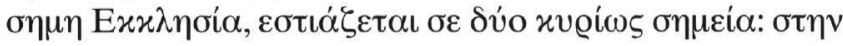

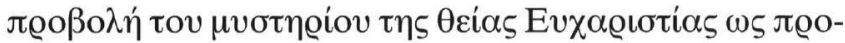

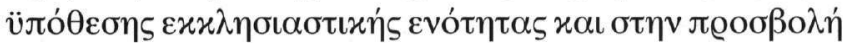

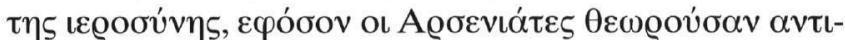

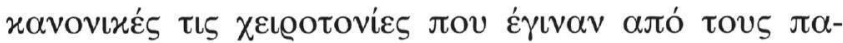

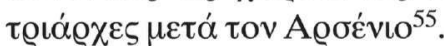

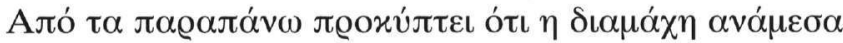

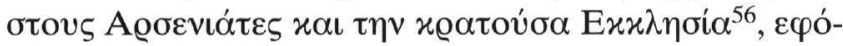

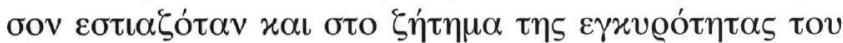

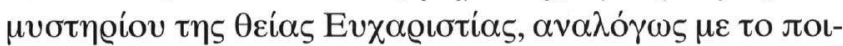

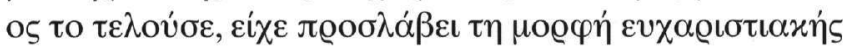

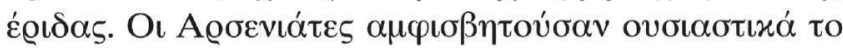

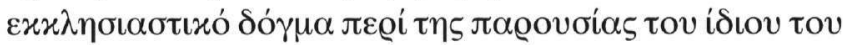

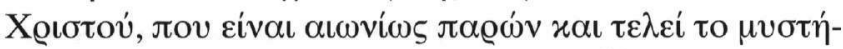

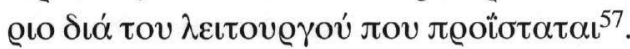

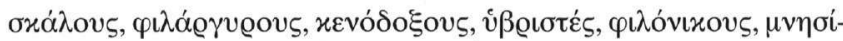

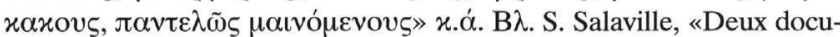
ments inédits sur les dissensions religieuses byzantines entre 1275 et 1310», REB 5 (1947), 122.

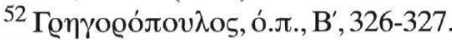

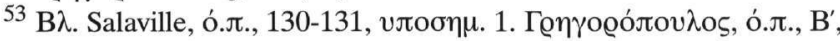
329-330.

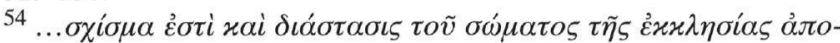

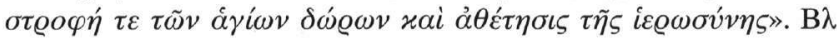

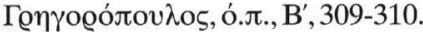

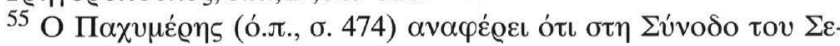

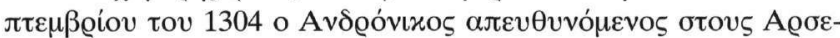

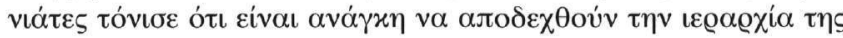

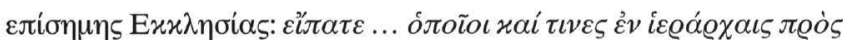

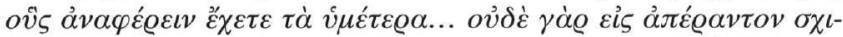

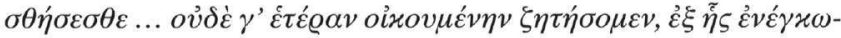

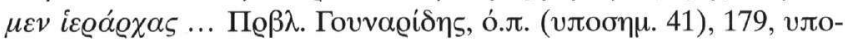
$\sigma \eta \mu .75$.

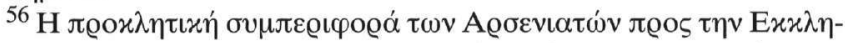

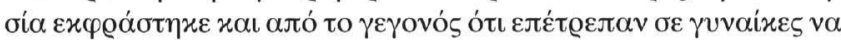

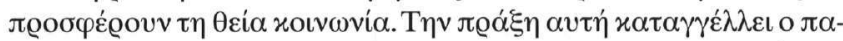

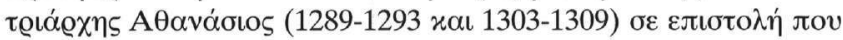

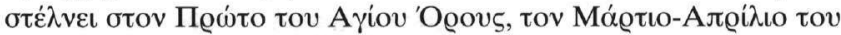

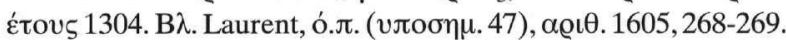

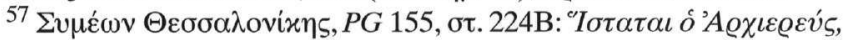

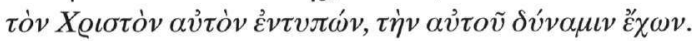




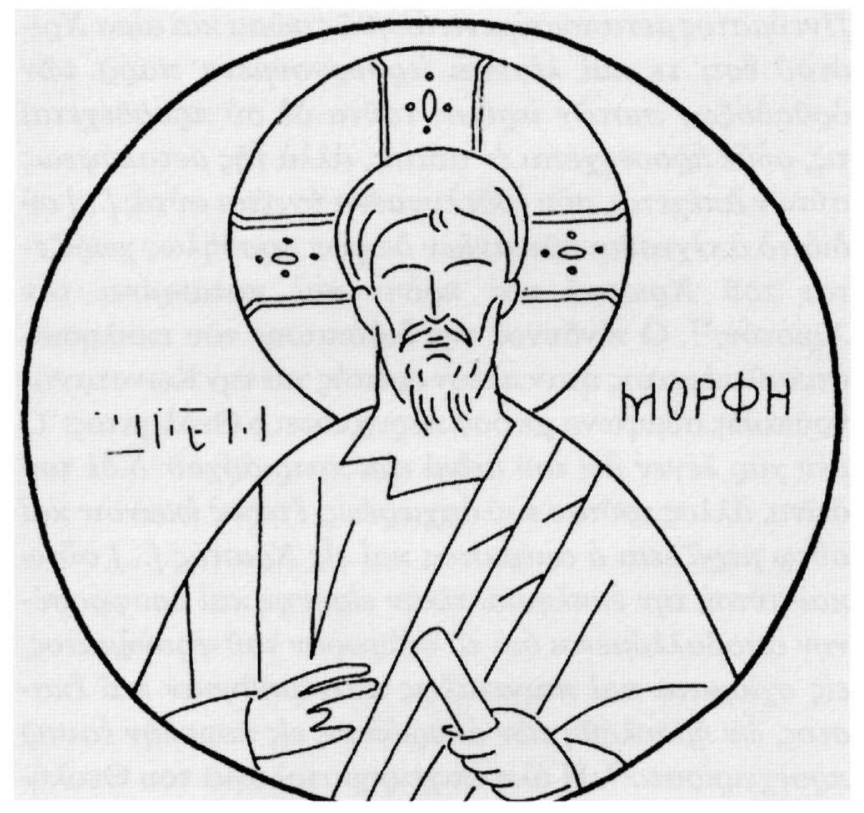

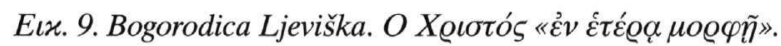

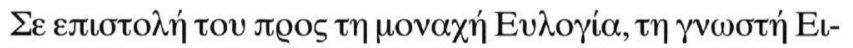

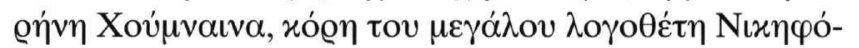

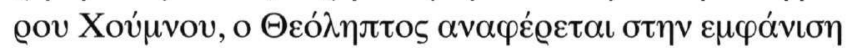

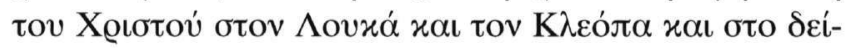

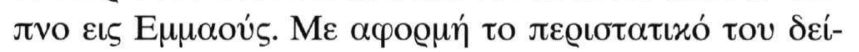

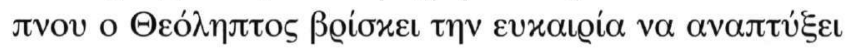
$\tau \eta \delta$

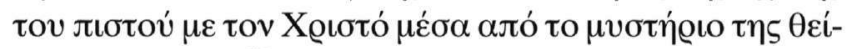

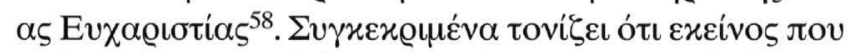

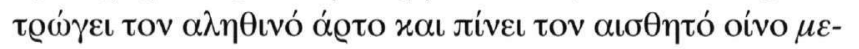

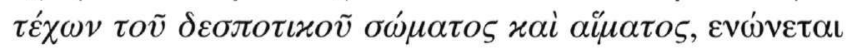

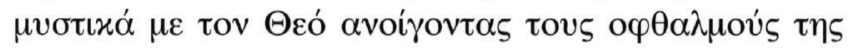

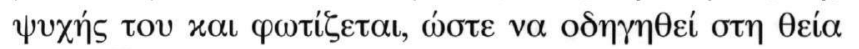

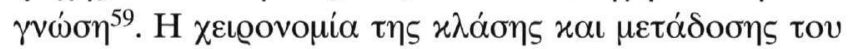

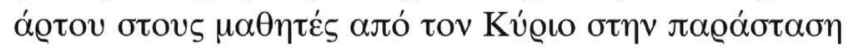

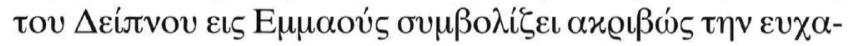

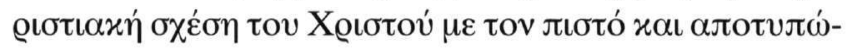

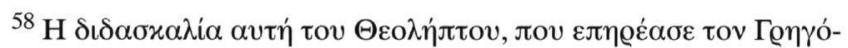

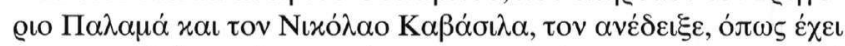

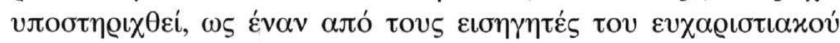

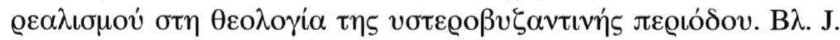
Meyendorff, «Spiritual Trends in Byzantium in the Late Thirteenth and

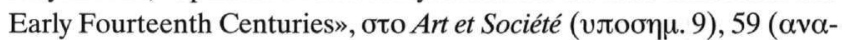

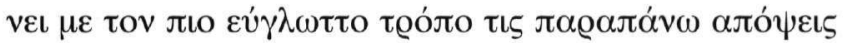

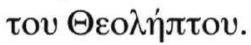

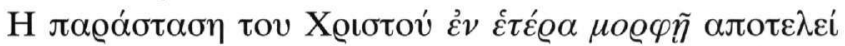

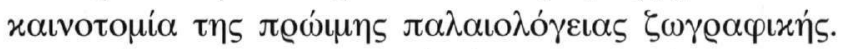

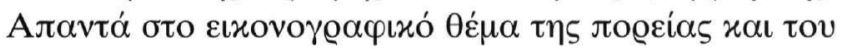

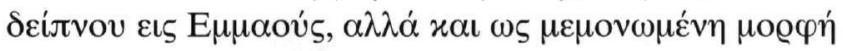

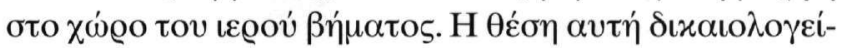

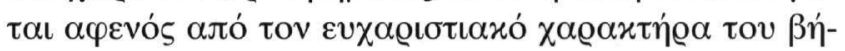

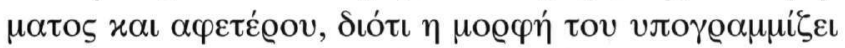

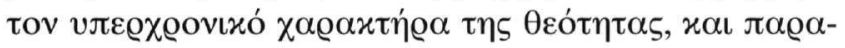

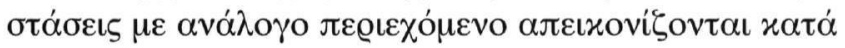

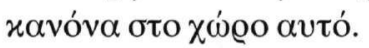

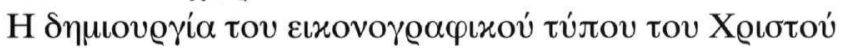

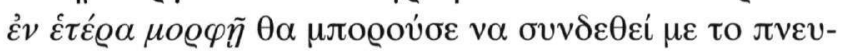

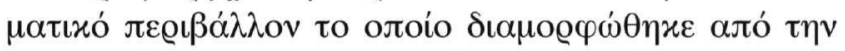

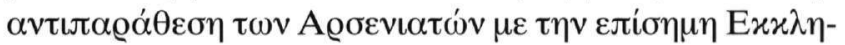

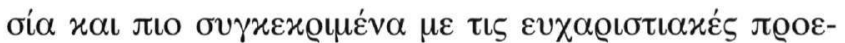

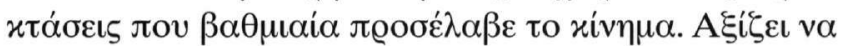

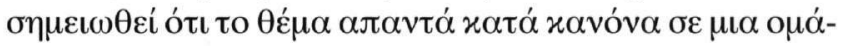

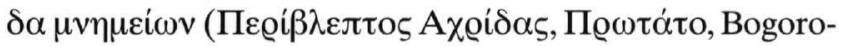

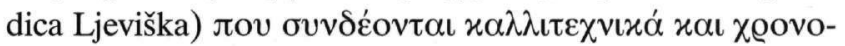

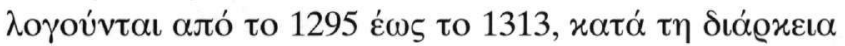

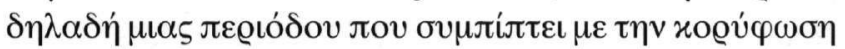

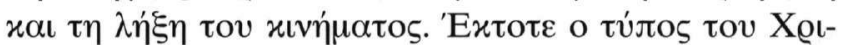

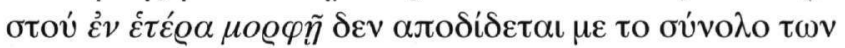

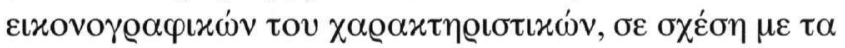

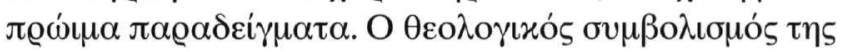

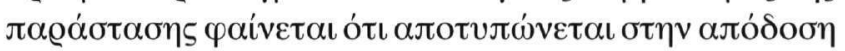

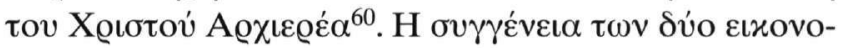

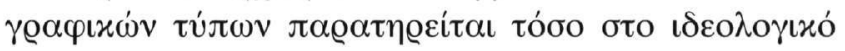

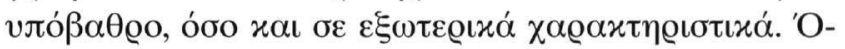

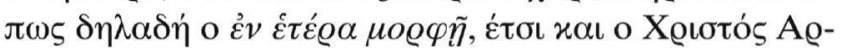

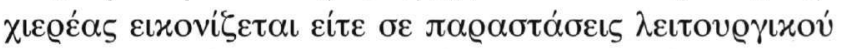

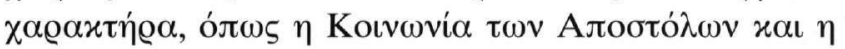

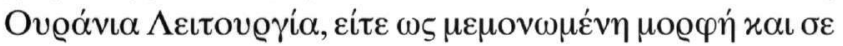

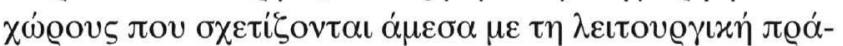

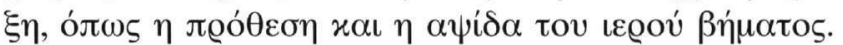

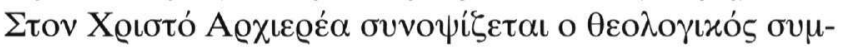

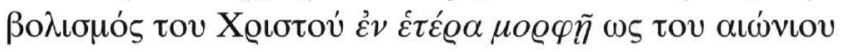

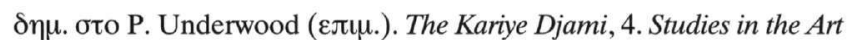
of the Kariye Djami, Princeton 1975,93-106.

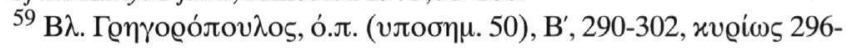
297.

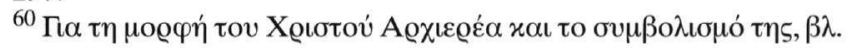

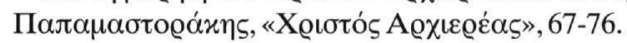




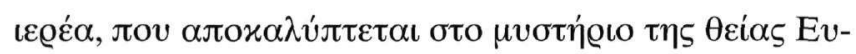

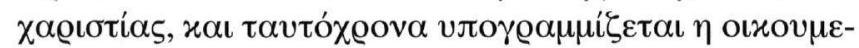

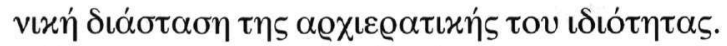

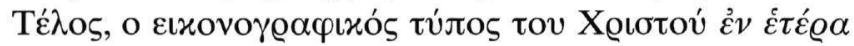

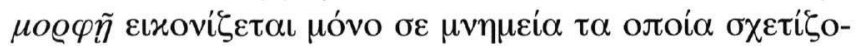

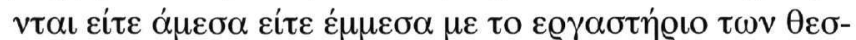

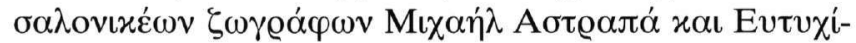

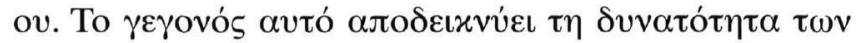

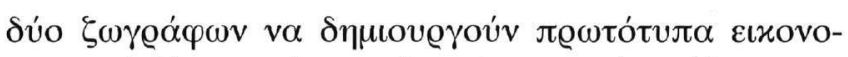

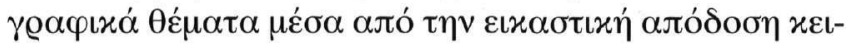

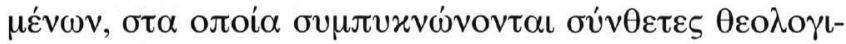

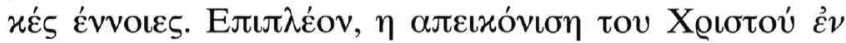

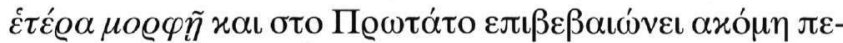

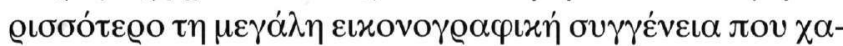

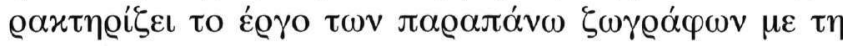

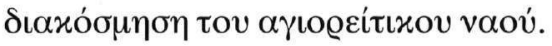

\section{Nektarios Zarras}

\section{CHRIST EN ETEPA MOPФH}

$\mathrm{T}_{\mathrm{r}}$

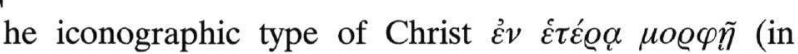
another form) derives from the pericope from the Gospel of St Luke (24:13-32), which describes the post-Resurrection appearance of Christ to Cleopas and to some other disciples in the course of their journey to the town of Emmaus, as well as at the supper that followed. Christ had not been recognized by his disciples until the hour of the repast, because according to the Gospel of St Mark (16:12), where the episode is mentioned briefly, the Lord had appeared to them "in another form". Cleopas' anonymous fellow traveller is identified in textual tradition with the Evangelist Luke, possibly because he is the only one who describes the incident in detail.

In the iconographic type of the $\varepsilon \dot{v} \varepsilon \dot{\varepsilon} \varepsilon \propto \alpha \mu o \varrho \varphi \tilde{\eta}$ Christ is represented which short hair, to the ears, a short beard and epikouris (tonsure). The use of this type in representations of the journey to and the supper at Emmaus, which belong to the cycle of Christ's appearances after the Resurrection, as well as the rendering of the Lord as a single figure with the inscription EN ETEPA MOPФH is encountered for the first time in the Palaiologan period.

Christ $\mathcal{\varepsilon} v \mathcal{\varepsilon} \tau \varepsilon \dot{\varepsilon} \varrho \mu o \varrho \varphi \tilde{\eta}$ is depicted in the scene of the Supper at Emmaus in the Peribleptos at Ochrid (1295) (Fig. 1) and in representations of the journey and the supper at Bogoridica Ljeviška (1309-1313) (Figs 2-3). The depiction of the Lord with epikouris and the decoration of his chiton with potami, imitating that of prelatic sticharia, underline his priestly of- fice. At Staro Nagoričino (1309-1318) (Fig. 4) the physiognomic features of Christ are not rendered in the iconograph-

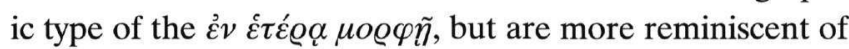
the usual type of a mature man. In the representations of the journey to and the supper at Emmaus in the Chilandari monastery (c. 1321) Christ has only the short hair and short beard, while at Gračanica (1319-1321) and Curtea de Argeș (1380-1385) he is shown with his usual portrait features. In the same scenes at Dečani (1345-1350) (Fig. 6) Christ is depicted with short hair and torsure, while at Kučevište (1330) he is represented in the supper scene as at Dečani and in the journey in the usual iconographic type.

In the representation of the supper in the Protaton on Mount Athos (c. 1300) the figure of Christ has been destroyed (Fig. 7). However, on the basis of the iconography it can be surmised that he was depicted with torsure and only with the chiton, part of the decoration of which is discernible, as in the Peribleptos and Bogoridica Ljeviška.

The earliest precisely dated depiction of Christ as a single figure with the inscription IC - XC O EN ETEPA MOPФH is in the half vault of the prothesis of the Protaton (Fig. 8). Christ is depicted again as a single figure $\mathcal{\varepsilon} v \varepsilon \varepsilon \tau \varepsilon \varrho \alpha \mu o \varrho \varphi \tilde{\eta}$ in the wall-paintings of St John Kaneo at Ochrid and the northwest cupola of Bogoridica Ljeviška (Fig. 9), which are almost contemporary with the Protaton.

Christ in the Supper at Emmaus is depicted either at the moment of breaking bread (Peribleptos at Ochrid, Staro 
Nagoričino), or at the moment of distributing the pieces to Luke and Cleopas (Bogoridica Ljeviška, Protaton, Dečani). The gesture of the breaking and the distributing of the bread, with its indisputable eucharistic symbolism, links the Supper at Emmaus semasiologically with the Last Supper. Christ in the representation of the Supper at Emmaus does not have a historical substance. His figure stresses his diachronic and mysterious presence through the celebration of the Holy Eucharist, in which the Resurrected Lord is eternally present and is mystically united with the faithful. In patristic literature the $\varepsilon \dot{v} \varepsilon \tau \dot{\varepsilon} \varrho \alpha \mu o \varrho \varphi \tilde{\eta}$ expresses essentially a theophany that transcends time and symbolizes Christ's state of divine glory after the Resurrection. The idea of the timeless character of the figure of Christ is emphasized by his physiognomic features. His youthful face surpasses the bounds of historical time and symbolizes the imperishability and the eternal beauty of the deity.

The representation of Christ $\dot{\varepsilon} v \dot{\varepsilon} \tau \varepsilon \dot{\varepsilon} \alpha \mu o \varrho \varphi \tilde{\eta}$ constitutes an innovation in Early Palaiologan painting. The appearance of this iconographic subject in the reign of Andronikos II Palaiologos (1282-1328) is correlated with the eucharistic problem, which was created by the Arseniates movement.
The Arseniates broke away from the official Church and created a separate religious community, because they doubted the authenticity of the ordinations made by the incumbents of the patriarchal throne after the anti-canonical defrocking of Patriarch Arsenius. The eucharistic symbolism of Christ $\dot{\varepsilon} v \dot{\varepsilon} \tau \varepsilon \dot{\varepsilon} \propto \mu o \varrho \varphi \tilde{\eta}$ is imprinted in the Hermeneia of Theoliptos of Philadelpheia (1250-1322), who takes the supper at Emmaus as predicate for developing his teaching on the authenticity of the relation between the believer and Christ through the mystery of the Holy Eucharist.

It is noteworthy that the subject is encountered as a rule in a group of monuments (Peribleptos, Protaton, Bogoridica Ljeviška) that are linked artistically and are dated between 1295 and 1313, a period coinciding with the end of the movement. From then on the type of Christ $\dot{\varepsilon} v \dot{\varepsilon} \tau \varepsilon \dot{\varepsilon} \alpha \mu \circ \varrho \varphi \tilde{\eta}$ is not portrayed with the whole set of its iconographic characteristics in relation to the early examples. The theological symbolism of this iconographic subject seems to be synopsized by the representation of Christ High Priest, which at once projects him as the eternal priest and enhances the ecumenical dimension of his prelacy. 